\title{
Absence of Stat1 in donor CD4+ T cells promotes the expansion of Tregs and reduces graft-versus-host disease in mice
}

\author{
Huihui Ma, ${ }^{1}$ Caisheng Lu, ${ }^{1}$ Judith Ziegler, ${ }^{1}$ Ailing Liu, ${ }^{1}$ Antonia Sepulveda, ${ }^{2}$ Hideho Okada, ${ }^{3}$ \\ Suzanne Lentzsch, ${ }^{1}$ and Markus Y. Mapara ${ }^{1}$
}

1Department of Medicine, Division of Hematology Oncology, Hematologic Malignancies Program, University of Pittsburgh Cancer Institute, Pittsburgh, Pennsylvania, USA. ${ }^{2}$ Department of Pathology and Laboratory Medicine, University of Pennsylvania, Philadelphia, Pennsylvania, USA. ${ }^{3}$ Department of Neurological Surgery, Brain Tumor Program, University of Pittsburgh Cancer Institute, Pittsburgh, Pennsylvania, USA.

\begin{abstract}
STAT1 is the main signal transducer for type I and II IFNs and plays a central role in the regulation of innate and adaptive immune responses. We used Stat1-deficient mice to test the role of donor Stat1 in MHC-matched minor histocompatibility antigen-mismatched (mHA-mismatched) and fully MHC-mismatched models of bone marrow transplantation. Lack of Stat1 in donor splenocytes reduced graft-versus-host disease (GVHD) in both immunogenetic disparities, leading to substantially attenuated morbidity and mortality. Donor Stat1 deficiency resulted in reduced alloantigen-induced activation and expansion of donor $T$ cells and correlated with the expansion of $\mathrm{CD}^{+} \mathrm{CD}^{+} 5^{+} \mathrm{Foxp}^{+}$Tregs in vivo. This expansion of Tregs was further confirmed by studies showing that Stat1 deficiency promoted the proliferation, while inhibiting the apoptosis, of natural Tregs, and that absence of Stat 1 enhanced the induction of inducible Tregs both in vitro and in vivo. Ex vivo expanded Stat $1^{-/-}$Tregs were superior to wild-type Tregs in suppressing alloantigen-driven expansion of $T$ cells in vitro and in inhibiting the development of GVHD. These observations demonstrate that Stat 1 is a regulator of Tregs and that targeting Stat 1 in $\mathrm{CD}^{+} \mathrm{T}$ cells may facilitate in vitro and in vivo expansion of Tregs for therapeutic use.
\end{abstract}

\section{Introduction}

Allogeneic bone marrow transplantation (BMT) has the potential to cure a number of benign (1-3) and malignant hematological disorders $(4,5)$. However, development of graft-versus-host disease (GVHD) is the most serious clinical problem limiting the widespread application of allogeneic BMT (6). Current paradigms in its pathobiology suggest that acute GVHD occurs through a complex interplay between host APCs (7), donor T cells, and conditioninginduced cytokines/chemokines $(6,8,9)$.

IFN- $\gamma$ is a key cytokine regulating innate and adaptive immune responses, with pleiotropic functions acting on macrophages, T cells, and NK cells (10). Its role in the development of GVHD has remained enigmatic, as IFN- $\gamma$ has the ability to promote or attenuate GVHD depending on the experimental conditions (11-15). Actions enhancing GVHD include direct cytopathic effects on the gastrointestinal epithelium (16) and promotion of Th1 differentiation (17), while GVHD inhibitory effects include the induction of activation-induced cell death (AICD) in donor T cells $(14,18)$. In contrast, very few studies have been published to date on the effects of type I IFNs on the pathogenesis of GVHD (19-21). Type I IFNs (e.g., IFN- $\alpha$ ) are a critical link to the innate and adaptive immune responses. Their early induction by various pathogen-associated molecular patterns, such as viruses, tumors, and apoptotic cells, provides one of the most important priming mechanisms for the subsequent establishment of adaptive immunity (22).

Authorship note: Huihui Ma and Caisheng Lu are co-first authors and contributed equally to this work.

Conflict of interest: Suzanne Lentzsch receives research funding from Celgene and serves as consultant for Celgene. Markus Y. Mapara received research funding from Resolvyx Inc. and holds Gentium stocks.

Citation for this article: J Clin Invest. 2011;121(7):2554-2569. doi:10.1172/JCI43706
Stat 1 is the main signal transducer for both type I and type II IFNs (23). Recent studies revealed that Th1 cell development is initiated by Stat 1 activation in response to IFN- $\gamma$ stimulation $(24,25)$. During T cell activation T-bet is induced by IFN- $\gamma$ and STAT1 signaling $(24,25)$. Once triggered, T-bet in turn activates IFN- $\gamma$ expression, leading to autocrine and paracrine positive feedback effects on IFN- $\gamma /$ Stat 1 signaling and Th1 differentiation $(24,25)$. We previously demonstrated that activation of Stat 1 in GVHD target tissue and secondary lymphoid organs is one of the earliest events in the induction of GVHD (26).

We therefore hypothesized that Stat 1 in donor CD4 T cells is a regulator of acute GVHD. Indeed, as we show here, we found that donor Stat 1 deficiency significantly inhibited minor histocompatibility antigen-mismatched (mHA-mismatched) GVHD and reduced GVHD in a fully MHC-mismatched setting. Protection from GVHD in recipients of Stat1-deficient splenocytes was associated with impaired Th1 differentiation and markedly enhanced expansion of functional Treg populations. Conversely, ex vivo expanded Stat1 $1^{-/}$Tregs significantly suppressed the induction of GVHD. Furthermore, we observed enhanced proliferation and resistance to cell death of Stat1-deficient Tregs; blocking IFN- $\gamma$ yielded similar results. Together, these findings support a physiological role for Stat 1 in the suppression of Treg generation, the promotion of AICD, and facilitation of Th1 differentiation during GVHD.

\section{Results}

Absence of Stat1 in donor spleen cells leads to reduced GVHD in both MHC-matched mHA-mismatched and fully MHC-mismatched recipients. To examine the role of donor Stat 1 in regulating development of GVHD, we induced GVHD in MHC-matched mHA-mismatched B6 $\left(\mathrm{H} 2^{\mathrm{b}}\right)$ and fully MHC-mismatched BALB/c $\left(\mathrm{H} 2^{\mathrm{d}}\right)$ mice by using Stat $1^{+/+}$or Stat $1^{-/-} 129 \mathrm{~S} 6 / \mathrm{SvEv}\left(\mathrm{H} 2^{\mathrm{b}}\right)$ donors. First we investigated 

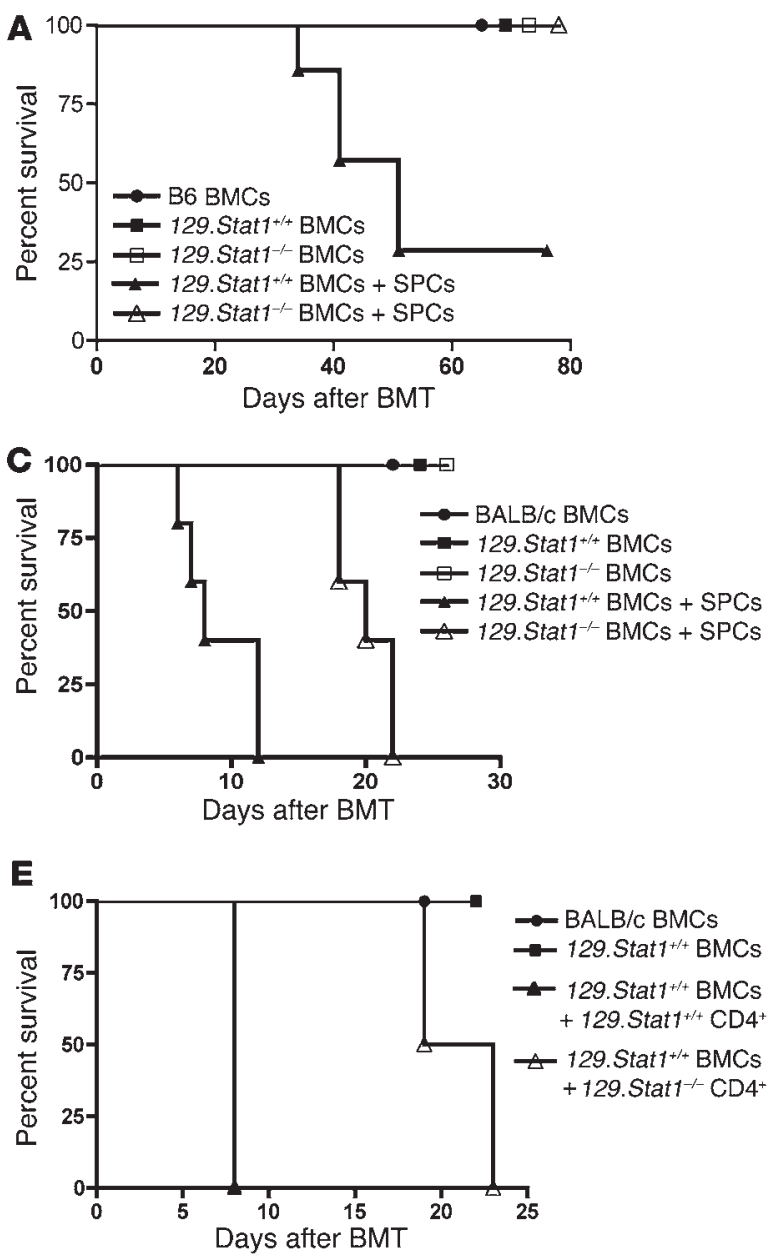

Figure 1

Stat1-deficiency in donor spleen cells leads to reduced GVHD. (A) mHA-mismatched GVHD. B6 mice received $5 \times 10^{6}$ BMCs and $4 \times 10^{7}$ splenocytes (SPCs) from 129 . Stat $1^{-/-}$or 129 . Stat $1^{+/+}$mice. B6 mice receiving $B M C$ s from $B 6,129$. Stat $1^{+/+}$, or 129 . Stat $1^{-/-}$mice were used as controls. Recipients of 129. Stat $1^{-1}$ BMCs and splenocytes were protected from GVHD-induced mortality compared with wildtype recipients (MST not reached versus 51-day MST; $P=0.023$ ). (B) Clinical GVHD scores on days 6 and 30 following mHA-mismatched BMT. Data are representative of 1 of 2 independent experiments ( $n=6-7 /$ group). (C) Fully MHC-mismatched GVHD (129Sv[H2b] to BALB/c [H2d]) strain combination using 129. Stat $1^{-/-}$or 129 . Stat $1^{+/+}$splenocytes. BALB/c mice receiving BMCs from BALB/c, 129. Stat $1^{+/+}$, or 129. Stat1 $1^{-/-}$mice were used as controls. (D) Clinical GVHD scores on day 6 after MHC-mismatched BMT. Data are representative of 1 of 3 similar experiments ( $n=5-10 /$ group). (E) Delayed mortality following GVHD induction in BALB/c recipients using purified CD4+ ${ }^{+}$cells $\left(3 \times 10^{6}\right)$ from 129. Stat1 $1^{+/+}$or 129. Stat $1^{-/-}$mice and BMT with $5 \times 10^{6}$ TCD Stat1 $1^{+/+}$BMCs. Recipients of BALB/C or 129 . Stat1+/+ BMCs were used as controls. Mortality was significantly delayed in recipients of 129. Stat1-- CD4+ T cells (MST 7.5 days versus 21 days; $P=0.037$ ). (F) Clinical GVHD score on day 6 after BMT. Data are representative of 1 of 2 similar experiments ( $n=5 / \mathrm{group}$ ). Clinical scores for individual animals are shown in B, D, and F. Horizontal bars denote the mean of clinical scores.

the role of donor Stat 1 in the clinically relevant mHA-mismatched strain combination. GVHD was induced in B6 mice following lethal irradiation with 1,075 cGy using $5 \times 10^{6} \mathrm{~T}$ cell-depleted (TCD) bone marrow cells (BMCs) and $4 \times 10^{7}$ splenocytes from either Stat $1^{+/+}$ or Stat $1^{-/-}$mice. Lack of Stat 1 led to complete protection from GVHD-induced mortality compared with recipients of wild-type splenocytes (median survival time [MST] 51 days; log-rank test, $P<0.005$; Figure 1A) and to reduced morbidity as demonstrated by significantly reduced weight loss (Supplemental Figure 1;

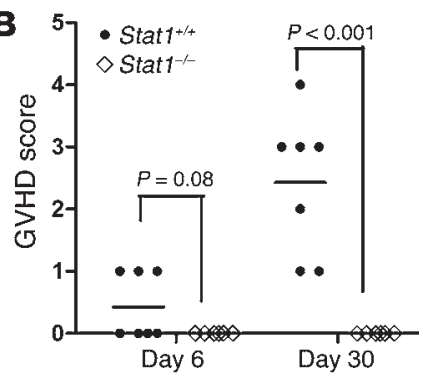

supplemental material available online with this article; doi:10.1172/ JCI43706DS1) and lower clinical GVHD score (Figure 1B).

Next we addressed the question of whether Stat 1 deficiency would also affect survival in a more aggressive, fully MHC-mismatched model of GVHD. BALB/c recipients $\left(\mathrm{H} 2^{\mathrm{d}}\right)$ were lethally irradiated with $800 \mathrm{cGy}$ and reconstituted with $5 \times 10^{6}$ allogeneic BMCs and $5 \times 10^{6}$ spleen cells from either 129. Stat $^{+/+}$or 129 . Stat $1^{-/-}$ donors $\left(\mathrm{H} 2^{\mathrm{b}}\right)$. As shown in Figure $1 \mathrm{C}$, recipients of Stat $1^{-/-}$splenocytes had significantly delayed GVHD mortality (MST 20 days versus 8 days; $P=0.004)$ and GVHD morbidity (clinical GVHD score on day 6 following BMT: $0.50 \pm 0.15$ versus $3.10 \pm 0.18$; $P<0.0001$; Figure 1D) compared with recipients of Stat $1^{+/+}$splenocytes.

As engraftment of Stat1-deficient cells may influence the induction of GVHD, we assessed the effects of Stat 1 deficiency on donor cell engraftment in the bone marrow and spleen. However, we did not see any significant differences in donor cell chimerism in $\mathrm{B} 220^{+}, \mathrm{Mac1}^{+}, \mathrm{CD}^{+}$, or $\mathrm{CD}^{+}$cells (Supplemental Figure 2).

In the fully MHC-mismatched 129 to $\mathrm{BALB} / \mathrm{c}$ model of acute GVHD, mortality is primarily CD4 dependent, similar to other wellestablished fully MHC-mismatched GVHD models (27). To exclude the contribution of donor bone marrowderived Stat1 $1^{-/}$APCs in modulating development of GVHD as described by Capitini et al. (28), lethally irradiated BALB/c mice were reconstituted with $5 \times 10^{6} 129$. Stat $1^{+/+}$TCD BMCs and co-injected with purified $3 \times 10^{6}$ splenic $\mathrm{CD}^{+} \mathrm{T}$ cells from Stat $1^{+/+}$or Stat $1^{-/-}$mice. Similar to recipients of Stat1-deficient splenocytes, significantly extended MST was observed in recipients of purified Stat1-deficient $\mathrm{CD}^{+} \mathrm{T}$ cells (MST 23 days versus 8 days; $P=0.037$; Figure $1 \mathrm{E})$. In additional experiments, we determined that the $T$ cell inoculum rather than the Stat 1 status of BMCs was responsible for the reduced ability to induce GVHD (data not shown).

Furthermore, GVHD-associated organ damage was assessed in recipients of Stat $1^{-/-}$and Stat $1^{+/+}$whole splenocytes in the mHAmismatched and MHC-mismatched setting (Figure 2) on day 6 following BMT. Significantly reduced GVHD target organ damage was observed in liver, small intestine, and colon in both immunogenetic disparities. We noted, however, that in the MHC-mis- 

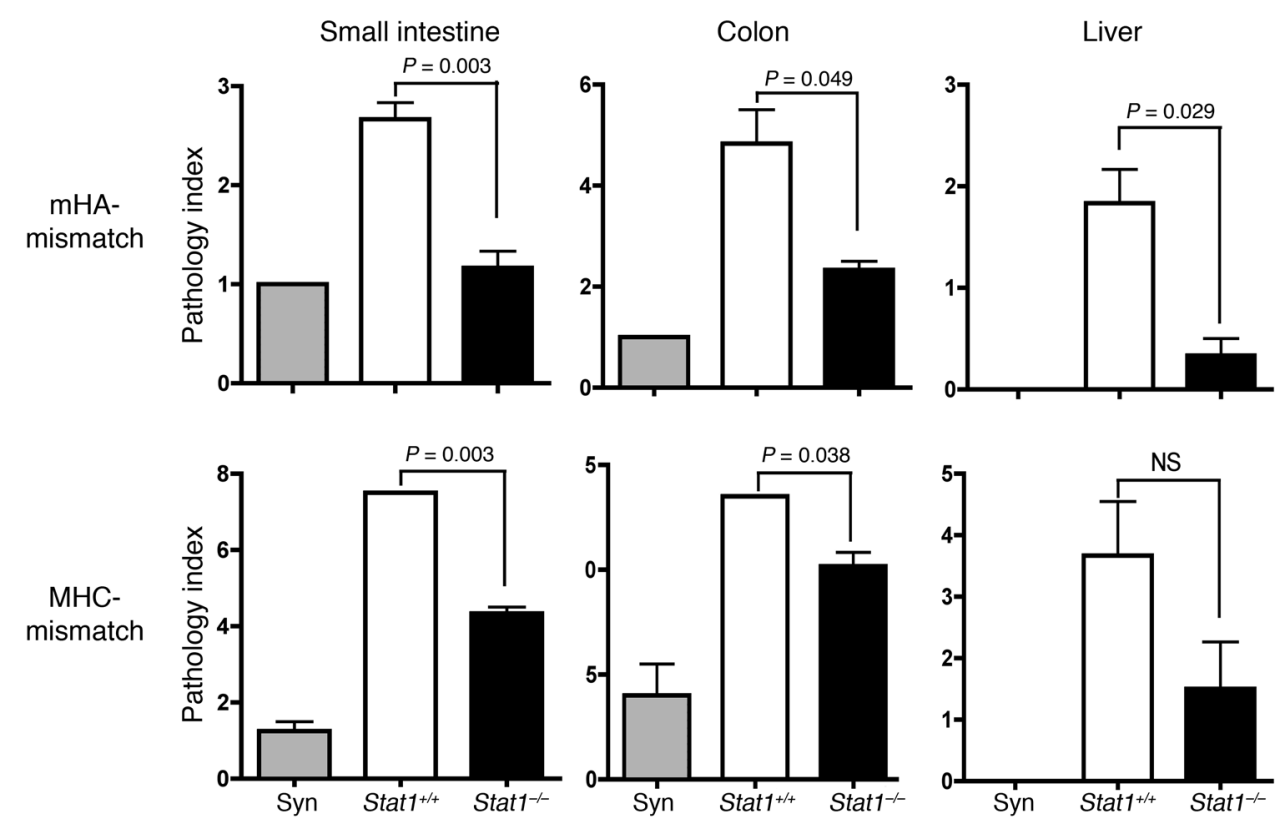

\section{Figure 2}

Reduced GVHD histopathology in recipients of Stat1-deficient donor cells. GVHD-associated tissue damage was assessed in small intestine, colon, and liver samples from recipients of syngeneic (Syn), Stat $1^{+/+}$, or Stat1-/- BMCs plus splenocytes following $\mathrm{mHA}$-mismatched or fully MHC-mismatched transplantation. Tissue samples were collected on day 6 after BMT. Data are representative of 1 of 2 similar experiments with 3-5 animals per group. matched setting, recipients of Stat $1^{-/-}$splenocytes eventually developed clear clinical and histopathological signs of GVHD (Supplemental Figure 3, A and B). We also observed increased donor $\mathrm{T}$ cell activation $\left(\mathrm{CD} 44^{+} \mathrm{CD} 62 \mathrm{~L}^{-}\right.$cells on day 14 compared with day 6 following BMT; Supplemental Figure 3C), and these cells were host reactive (Supplemental Figure 3D), confirming that in the MHC-mismatched setting, animals succumbed to GVHD rather than infection or non-GVHD-related causes.

Absence of Stat1 leads to reduced activation and expansion of donor lymphocytes. Recent studies have shown that type I IFN signaling contributes to the expansion of $\mathrm{CD}^{+} \mathrm{T}$ cells in response to viral infections in vivo (29). Furthermore, IFN- $\gamma$ has also been suggested to play a role in maintaining immune homeostasis through induction of AICD in clonally expanded CD4 $4^{+} \mathrm{T}$ cells (30). Thus, IFN- $\gamma-$ deficient donors have been reported to induce more significant GVHD $(18,30)$, which is partly attributable to the reduced AICD of alloantigen-activated T cells (31). However, little is known about the role of Stat 1 and the shared type I and type II IFN signaling pathway in regulating activation, expansion, and contraction of donor lymphocytes in the scenario of GVHD, although it has been suggested that donor cells lacking the IFN- $\gamma$ receptor induce less GVHD (17). As a first step, we studied the effects of Stat 1 deficiency on the in vivo expansion of donor $T$ cells following allogeneic BMT. Compared with Stat $1^{+/+}$splenocytes, we observed reduced expansion of both donor $\mathrm{CD}^{+}$and $\mathrm{CD}^{+}$Stat $1^{-/-}$lymphocytes in both mHA-mismatched and MHC-mismatched GVHD models (Figure 3A and Supplemental Figure 4).

Several mechanisms might account for the decreased numbers of Stat1-deficient donor $\mathrm{CD}^{+}$and $\mathrm{CD}^{+} \mathrm{T}$ cells (e.g., impaired activation, reduced proliferation, or increased cell death) after infusion into recipients. We therefore studied alloantigen-induced activation and proliferation of donor T cells following allogeneic BMT. To distinguish between donor and host cells in the mHA-mismatched setting, we used congenic B6.SJL $\left(\mathrm{H}-2^{\mathrm{b}}, \mathrm{CD} 45.1^{+}\right)$mice as recipients. To determine activation and proliferation of donor $\mathrm{CD}^{+}$and $\mathrm{CD}^{+}{ }^{+} \mathrm{T}$ cells, CD45.2 $2^{+} 129$. Stat $^{1^{+/+}}$and 129. Stat $^{1^{-/-}}$donor cells were labeled with CFSE and injected. Mice were sacrificed, and spleens were harvested on day 6 after BMT. As illustrated in Figure 3B, donor Stat $^{-/-} \mathrm{CD}^{+} \mathrm{T}$ cells showed significantly reduced cell division and proliferation (30\% $\mathrm{CFSE}^{\text {lo }}$ in Stat $1^{-\gamma_{-}} \mathrm{CD}^{+} \mathrm{T}$ cells

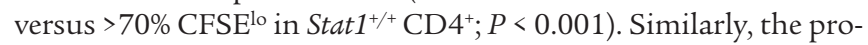
liferation of donor Stat1 $1^{-/} \mathrm{CD}^{+}$cells was also reduced compared with $\mathrm{Stat}^{+/+} \mathrm{CD}^{+}$cells (Figure 3B). When donor $\mathrm{CD} 4^{+}$and $\mathrm{CD} 8^{+}$ $\mathrm{T}$ cells were analyzed for expression of CD62L and CD69, recipients of Stat1 $1^{-/}$cells showed significantly reduced expression of CD69 while lacking activation-induced downregulation of $\mathrm{CD} 62 \mathrm{~L}$ on donor $\mathrm{CD}^{+}$and $\mathrm{CD}^{+} \mathrm{T}$ cells (Figure $4 \mathrm{~A}$ ). In fact, the expression of CD62L on Stat1-deficient $\mathrm{CD}^{+}$and $\mathrm{CD}^{+} \mathrm{T}$ cells was comparable to untreated controls and higher compared with syngeneic recipients (Figure 4A; $P<0.05$ ), suggesting that conditioning-induced $T$ cell activation was sufficient for CD62L downregulation in syngeneic recipients. To further study the relationship between proliferation and activation in donor lymphocytes, CD69 and CD62L expression was also tested in relation to CFSE dilution. As shown in Figure 4B, Stat 1 deficiency resulted in significantly reduced activation and proliferation, as indicated by the reduced expression of $\mathrm{CD} 69$, the retention of CD62L expression, and the finding that the majority of cells were CFSEhi.

We next evaluated CD25 antigen expression on donor T cells following BMT. In the MHC-matched $\mathrm{mHA}$-mismatched setting, we observed a significant decrease in CD25 expression on donor $\mathrm{CD}^{+}$and $\mathrm{CD}^{+}$cells in recipients of Stat $1^{-/-}$splenocytes on day 6 after BMT compared with that of Stat $1^{+/+}$. Of note, the CD $25^{+}$ population was also $\mathrm{CFSE}^{\text {lo }}$, indicating rapid proliferation (Figure 4C). IFN- $\gamma$ is well known for its role in promoting AICD. Thus, the absence of Stat 1 signaling may block induction of apoptosis in donor lymphocytes following allogeneic BMT. To monitor the induction of apoptosis and its association with activation and proliferation, we determined Annexin V expression in donor lymphocytes in relation to CD25 expression and CFSE dilution. As shown in Figure 4D, there was significantly increased apoptosis in Stat $1^{+/+}$ $\mathrm{CD}^{+}$and $\mathrm{CD} 8^{+}$lymphocytes compared with Stat1-deficient cells. This difference between Stat $1^{+/+}$and Stat $1^{-/-} \mathrm{CD} 4^{+}$and $\mathrm{CD}^{+} \mathrm{T}$ cells was most pronounced in the $\mathrm{CD} 25^{+} \mathrm{CFSE}^{\text {lo }}$ fraction, while there 
A

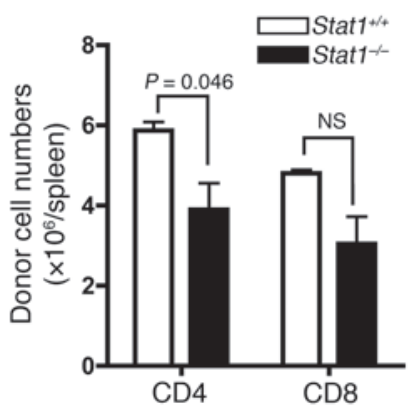

B

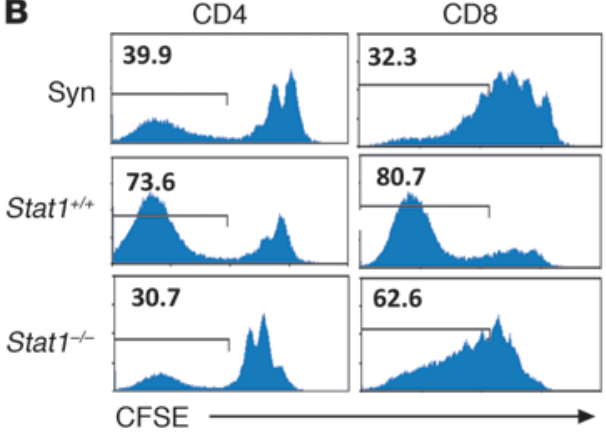

Figure 3

Absence of Stat1 in donor lymphocytes leads to reduced expansion of donor lymphocytes in mHA-mismatched allogeneic BMT. GVHD was induced in lethally irradiated B6.SJL mice. Recipients were reconstituted with TCD $5 \times 10^{6} 129$. Stat $1^{+/+}$ BMCs together with $4 \times 10^{7}$ CFSE-labeled 129 . Stat $1^{+/+}$or 129 . Stat $1^{-/-}$splenocytes. On day 6 after BMT, animals were sacrificed and splenocytes analyzed by FCM. Representative results are shown from 1 of 2 independent experiments ( $n=3 / g r o u p)$. (A) Donor-derived $\left(C D 45.2^{+}\right) \mathrm{CD}^{+}$and $\mathrm{CD} 8^{+}$cells were detected and absolute numbers presented. (B) In vivo proliferation of donor $\mathrm{CD}^{+}$and $\mathrm{CD} 8^{+} \mathrm{T}$ cells was assessed by CFSE dilution detected by FCM. Data in graph are mean \pm SEM.

was no difference in the $\mathrm{CD}^{-} 5^{-} \mathrm{CFSE}^{\text {hi }}$ population (i.e., nonactivated, nonproliferating cells; Figure 4D).

In the MHC-mismatched model setting, we also noted reduced expansion of Stat 1 -deficient donor $\mathrm{CD}^{+}$and $\mathrm{CD}^{+}$cells $(P=0.09$ and $P=0.03$ ) (Supplemental Figure 4A). Again, Stat 1 deficiency resulted in reduced cell division and proliferation, with a significantly increased $\mathrm{CFSE}^{\text {hi }}$ cell population. However, both donor $\mathrm{CD}^{+}$and $\mathrm{CD}^{+}$cells proliferated much more vigorously (compared with the mHA-mismatched setting), and more than $90 \%$ of cells became CFSElo (Supplemental Figure 4, B and D). In contrast to the mHA-mismatched setting and compared with their Stat $1^{+/+}$counterparts, CD25 expression was not reduced but was even higher $(n=3 ; P=0.04)$ in donor Stat $1^{-/}$CD $4^{+}$cells (Supplemental Figure 4C). CD25 expression was almost exclusively restricted to the CFSElo cell population (Supplemental Figure 4D). Furthermore, we observed a significantly higher proportion of $\mathrm{CFSE}^{\text {hi }} \mathrm{CD}^{2} 5^{-}$cells (Supplemental Figure 4, B and D), and there were significantly lower $\mathrm{CD} 44^{+} \mathrm{CD} 62 \mathrm{~L}^{-}$cells within the Stat $1^{-/-} \mathrm{CD}^{+}$cell population (Supplemental Figure 4E), indicating that Stat 1 deficiency also led to reduced donor activation in the MHC-mismatched setting. Although we did not see a significant difference in apoptosis in the total donor CD4 cells, we did observe reduced Annexin $\mathrm{V}^{+}$cells in the activated Stat1 $1^{-/} \mathrm{CD} 25^{+}$ population (which was also $\mathrm{CFSE}^{\mathrm{lo}}$ ) compared with $\mathrm{Stat1}^{+/+}$ $\mathrm{CD} 25^{+}$cells (Supplemental Figure 4F), indicating that Stat 1 deficiency leads to reduced AICD.

The observation of decreased AICD following strong alloantigendriven TCR stimulation would lead to the expectation that alloreactive donor $\mathrm{T}$ cells should increase and not decrease over time. In vitro studies in fact confirmed that Stat1-deficient spleen cells or $\mathrm{CD}^{+}$cells from either Stat $1^{+/+}$or Stat $1^{-/-}$mice hyperproliferated in response to allostimulation. As shown in Supplemental Figure 5, both $S t a t 1^{-/}$spleen cells and $\mathrm{CD}^{+}$cells proliferated more vigorously than their $\operatorname{Stat} 1^{+/+}$counterparts. Given these discrepant in vitro findings, we hypothesized that the reduced expansion of alloreactive donor $\mathrm{T}$ cells, which we observed in vivo in both immunoge-

netic disparities, could not be entirely explained by an intrinsic defect of Stat1-deficient T cells.

Stat 1 deficiency in donor $T$ cells leads to skewed $C D 4^{+}$ $T$ cell differentiation. To further investigate the underlying mechanism of reduced GVHD in recipients of Stat1 ${ }^{-/-}$splenocytes, we studied the role of donor Stat 1 in controlling the systemic inflammatory response. For this purpose, we analyzed serum cytokine profiles in recipients of Stat $1^{-/-}$versus Stat $^{+/+}$donor cells on day 6 after BMT in the MHC-mismatched BMT and mHA-mismatched GVHD model. As shown in Figure 5A, lack of Stat1 in donor splenocytes led to significantly diminished Th1 cytokine levels (IFN- $\gamma$ : $508 \mathrm{pg} / \mathrm{ml}$ versus $84 \mathrm{pg} / \mathrm{ml}, P<0.05$; IL-12: $198 \mathrm{pg} / \mathrm{ml}$ versus 54 $\mathrm{pg} / \mathrm{ml}, P=0.08$; IL-15: $170 \mathrm{pg} / \mathrm{ml}$ versus $55 \mathrm{pg} / \mathrm{ml}$, $P=0.05)$ but significantly elevated IL-4 $(P=0.003)$, IL-5 $(P=0.007)$, and IL-17 $(P=0.03)$ levels in the fully MHC-mismatched strain setting. IL-6 levels were also increased, with a trend toward statistical significance $(P=0.08$, data not shown). Similar observations with skewed Th1/Th2 cytokine balance were made in the mHA-mismatched setting. We observed reduced TNF- $\alpha$, IFN- $\gamma$, CXCL9/MIG, and CXCL10/IP-10 but elevated IL-5 levels (Figure 5B). However, we did not see any significant difference in IL-12p70, IL-10, or IL-17 in this setting (Figure 5B), nor did we see any significant difference in IL-2 levels on day 6 after BMT in either the MHC- or mHA-mismatched settings (data not shown).

To confirm attenuated Th1 differentiation in the absence of Stat1, we studied Stat $1^{+/+}$and Stat $1^{-/-} \mathrm{CD}^{+} \mathrm{T}$ cells for their Th1 differentiation capacity in vitro and in vivo by analyzing IFN- $\gamma$ and T-bet expression, which are hallmarks of Th1 differentiation. Stat $1^{+/+}$and Stat1 $1^{-/}$splenic CD4 ${ }^{+} \mathrm{T}$ cells were stimulated with immobilized $\alpha$-CD3 and soluble $\alpha$-CD28 antibodies in the presence (Th1 conditions) or absence of murine IL-12 and anti-IL-4 antibody and studied for the induction of T-bet and IFN- $\gamma$ expression. Stat1-deficient $\mathrm{CD}^{+} \mathrm{T}$ cells were significantly impaired in their ability to convert to IFN- $\gamma$-producing Th 1 cells during in vitro cultivation (Figure 5C), indicating that even IL-12 could not bypass the block of Th1 differentiation in Stat1-deficient CD $4^{+}$ $\mathrm{T}$ cells. In line with the in vitro studies, donor Stat $1^{-/-} \mathrm{CD} 4^{+} \mathrm{T}$ cells displayed significantly decreased T-bet and IFN- $\gamma$ (Figure 5D) but increased IL-4 and IL-17 expression (Figure 5E) in vivo in the MHC mismatched setting on day 6 following BMT. We observed the same attenuated IFN- $\gamma$ expression in donor Stat $1^{-1-} \mathrm{CD} 4^{+}$cells on day 6 after BMT in the mHA-mismatch setting (data not shown).

Lack of Stat1 in donor CD4 T cells leads to increased Foxp $3^{+}$Tregs. Since Stat 1 deficiency in donor lymphocytes resulted in reduced GVHD and impaired Th1 differentiation, we were interested in whether inhibition of GVHD involved a Treg-dependent mechanism. On day 6 after BMT, we found a highly significant increase in the proportion of donor type $\mathrm{CD} 4^{+} \mathrm{CD} 25^{+} \mathrm{Foxp}^{+}$Tregs in recipients of Stat $1^{-/}$splenocytes compared with that in recipients of Stat $1^{+/+}$splenocytes in both immunogenetic disparities (MHCmismatched and MHC-matched mHA-mismatched setting; both $P<0.001$; Figure 6, A and B). In the mHA-mismatched setting, we observed that the absolute numbers of Tregs in the spleen from recipients of Stat $1^{-/-}$cells were significantly higher than those of Stat $1^{+/+}$cells (Figure 6B). However, we did not find significant dif- 
A

CD4

CD8

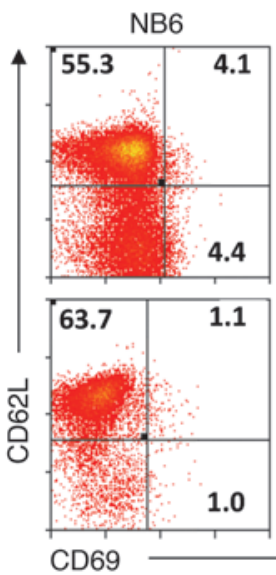

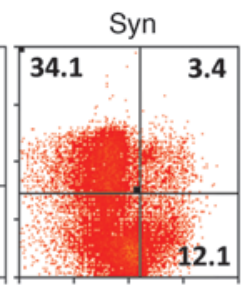
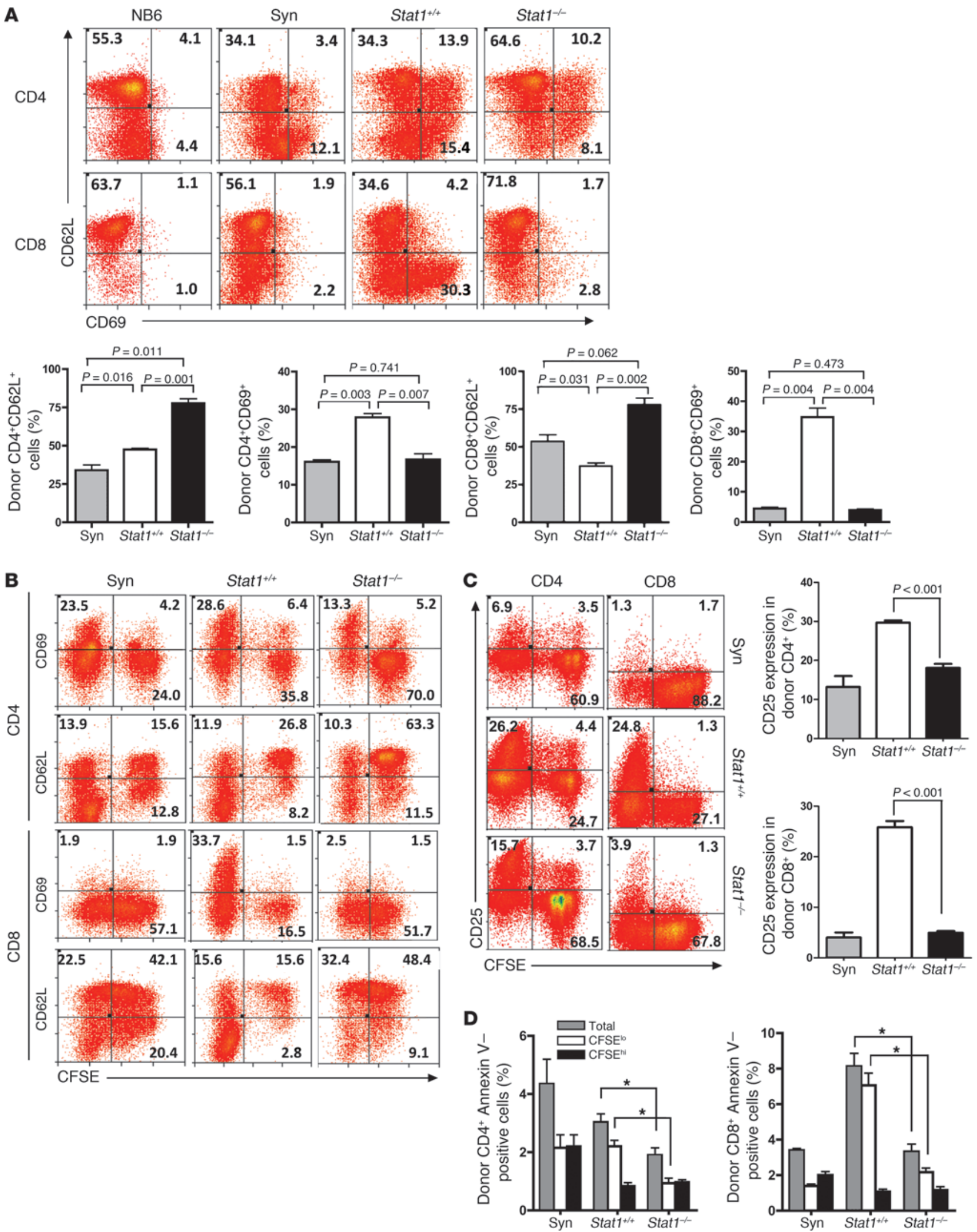


\section{Figure 4}

Absence of Stat1 in donor lymphocytes leads to reduced activation and activation induced cell death (AICD). On day 6 after BMT, animals were sacrificed and splenocytes were analyzed by FCM. (A) CD69 and $\mathrm{CD62L}$ expression was assessed in donor $\mathrm{CD}^{+}$or $\mathrm{CD}^{+}{ }^{+} \mathrm{T}$ cells from Stat $1^{+/+}$or Stat $1^{-/-}$mice and syngeneic controls. Normal B6 mice (NB6) were used as controls. Summary data of CD69 and CD62L expression are shown in the graphs. (B) CD69 and CD62L expression and (C) CD25 expression were analyzed in rapidly (CFSElo) and slowly (CFSE ${ }^{\text {hi) }}$ dividing donor $\mathrm{CD}^{+}$and $\mathrm{CD}^{+} \mathrm{T}$ cells. Numbers represent the percentages of cells present in the given quadrant. Summary data of $\mathrm{CD} 25$ expression in unfractionated donor $\mathrm{CD}^{+}$and $\mathrm{CD} 8^{+}$cells are shown to the right. (D) Assessment of in vivo AICD as determined by Annexin $\mathrm{V}$ staining in donor CD4+ (left) and CD8+ (right) T cells was analyzed in total, rapidly, and slowly dividing cells $\left({ }^{*} P<0.05\right.$ versus Stat $\left.1^{+/+}\right)$. Data in graphs are mean \pm SEM.

ferences in the absolute numbers of Tregs in the fully MHC-mismatched setting (Figure 6A).

There are 2 pathways for development of Tregs: natural Tregs (nTreg) are derived from the thymus, while inducible Tregs (iTreg) are generated in the periphery from $\mathrm{CD}^{+} \mathrm{CD} 25^{-} \mathrm{Foxp}^{-}$ cells under inflammatory conditions. Natural and inducible $\mathrm{CD}^{+}{ }^{+} \mathrm{CD} 25^{+}{ }^{-}$oxp $33^{+}$Tregs (nTregs and iTregs, respectively) play a pivotal role in immune regulation and controlling inflammatory conditions. Tregs have been reported to prevent or delay the onset of GVHD in animal models (32), and the presence of Tregs in GVHD target organs has been shown to correlate negatively with the severity of GVHD (33).

To exclude the possibility that the observed increased Treg population during induction of GVHD may be due to constitutively elevated numbers of Tregs in Stat1-deficient mice, we assessed the Treg content in thymi and spleens of 129. Stat $1^{+/+}$and 129 . Stat $1^{-/-}$mice. In accordance with a previous report (34), we noted a significant decrease in thymic and splenic Tregs of Stat $1^{-/-}$animals (spleen: $3.2 \% \pm 0.2 \%$ versus $2.4 \% \pm 0.1 \%, P=0.024$; thymi: $6.4 \% \pm 0.7 \%$ versus $3.7 \% \pm 0.2 \%, P=0.008$; Supplemental Figure 6) compared with wildtype animals under steady-state conditions.

Besides the reduced proliferation of $\mathrm{CD} 25^{+}$conventional $\mathrm{CD} 4^{+}$ $\mathrm{T}$ cells as we showed in Figures 3 and 4, several other mechanisms including enhanced proliferation and/or reduced apoptosis of Stat1-deficient nTreg and/or iTregs might also account for the increased Foxp3 ${ }^{+}$population within the Stat1-deficient $\mathrm{CD} 4{ }^{+} \mathrm{CD} 25^{+}$cells. We therefore sought to delineate whether Stat 1 controls the expansion of nTregs, the induction of iTregs, or both via regulation of proliferation and cell death.

Absence of Stat1 in nTregs leads to reduced cell death and enhanced proliferation in vitro. To better understand the influence of Stat 1 deficiency on the proliferation of nTregs, purified splenic Stat1deficient or Stat 1 wild-type $\mathrm{CD} 4{ }^{+} \mathrm{CD} 25^{+}$cells were labeled with CFSE, cultured on $\alpha$-CD3-coated plates in the presence of $\alpha$-CD28 antibodies and IL-2 for 3 days, and analyzed for proliferation and induction of apoptosis. More than $95 \%$ of $\mathrm{CD} 4^{+} \mathrm{CD} 25^{+}$cells were $\mathrm{Foxp}^{+}$at the initiation of the culture (data not shown). As shown in Figure 7A, we observed significantly increased expansion of Stat $1^{-/-} \mathrm{CD} 4{ }^{+} \mathrm{CD} 25^{+} \mathrm{Foxp}^{+}$Tregs compared with Stat $1^{+/+}$Tregs $(P<0.001)$. Half of the Stat $1^{+/+}$starting population underwent cell death, as determined by trypan blue staining after 3 days of in vitro culture, compared with only $10 \%$ of Stat $1^{-/-}$cells (Figure 7B). When cell proliferation was monitored by CFSE dilution, Stat 1 deficiency in $\mathrm{CD}^{+} \mathrm{CD} 25^{+} \mathrm{Foxp}^{+}$Tregs resulted in a significantly higher proportion of $\mathrm{CFSE}^{\text {lo }}$ cells, which indicated more vigorous proliferation, with $85 \%$ Foxp $^{+}$cells being CFSElo in Stat $1^{-/-}$compared with

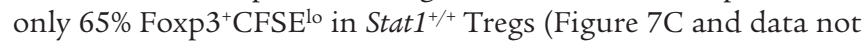
shown). Furthermore, at the end of the culture, $30 \%$ of the Stat $1^{+/+}$ $\mathrm{CD}^{+} \mathrm{CD} 25^{+}$population were Foxp3 $3^{-}$, compared with only $10 \%$ of the Stat $1^{-/-}$cells (Figure 7C). We suspected that these Foxp $3^{-}$cells may have converted from Foxp $3^{+}$Tregs upon strong TCR stimulation, as described by other investigators (35). Since TGF- $\beta 1$ has been reported to maintain Foxp3 expression and suppressor function of $\mathrm{CD} 4^{+} \mathrm{CD} 25^{+}$Tregs (36), we further analyzed whether this conversion could be inhibited by adding TGF- $\beta 1$ into the culture system. Indeed, TGF- $\beta 1$ almost completely abolished the generation of Foxp $3^{-}$cells (less than 5\%) in both Stat $1^{+/+}$and Stat $1^{-/-}$cells. However, Stat1-deficient cells proliferated more vigorously compared with Stat 1 wild-type cells, as indicated by the significantly higher proportion of CFSElo cells found in Stat $1^{-/-}$cells (>80\%) compared with Stat $1^{+/+}$cells $(<60 \%$; Figure $7 \mathrm{D})$, supporting the notion that lack of Stat 1 promotes in vitro expansion of nTregs.

IFN- $\gamma$ has been reported to induce apoptosis in many types of cells, including T cells (37). To understand the underlying mechanism of increased expansion of Stat $1^{-/-}$nTregs, we treated both Stat $1^{+/+}$and Stat $1^{-/-}$nTregs with $\alpha$-INF- $\gamma$ antibodies in the presence of IL- 2 and TGF- $\beta 1$. As shown in Figure 7E, blocking IFN- $\gamma$ by use of neutralizing antibodies significantly abrogated spontaneous induction of apoptosis of $\mathrm{Stat}^{+/+}$nTregs cultured under Treg conditions, indicating that autocrine/paracrine IFN- $\gamma$ inhibits Treg development. In contrast, we saw significantly lower levels of spontaneous apoptosis in Stat1-deficient Tregs, and the addition of $\alpha$-IFN- $\gamma$ antibodies did not further reduce apoptosis of these Stat1 $1^{-1}$ nTregs (Figure 7E), indicating that IFN- $\gamma /$ Stat 1 is a contributing factor for the induction of nTreg apoptosis under Treg conditions.

It is well established that the balance between p-Stat 1 and p-Stat 3 expression determines the fate of many cells. Thus, enhanced p-Stat 1 expression promotes induction of apoptosis and suppression of proliferation. In contrast, increased p-Stat 3 expression is associated with enhanced proliferation and reduced apoptosis (38, 39). To further elucidate the increased resistance to apoptosis of Stat1-deficient Tregs, we studied the expression and activation of Stat 1 and Stat 3 . In line with their increased resistance toward apoptosis, Stat 1 -deficient nTregs were found to have constitutively upregulated expression of p-Stat3 (Tyr705). Although there was no significant increase in Stat 3 expression or activation in wild-type nTregs following IFN- $\gamma$ neutralization, Stat 1 phosphorylation as well as total Stat 1 were significantly reduced, resulting in an overall reduction of the ratio of $\mathrm{p}$-Stat $1 / \mathrm{p}$-Stat 3 (Figure $7 \mathrm{~F}$ ).

Absence of Stat 1 in $\mathrm{CD}^{+} \mathrm{T}$ cells leads to enhanced in vitro and in vivo induction of iTregs in an IFN- $\gamma /$ Stat1-dependent way. We next determined the impact of Stat 1 deficiency on the generation of iTregs from $\mathrm{CD}^{+} \mathrm{CD} 25^{-}$cells. In vitro differentiation studies under Treg conditions (medium containing IL-2 and TGF- $\beta 1$ ) showed that induction of iTreg from Stat $1^{-/-} \mathrm{CD} 4^{+} \mathrm{CD} 25^{-}$cells was significantly enhanced compared with Stat $1^{+/+} \mathrm{CD} 4^{+} \mathrm{CD} 25^{-}$cells (Figure 8A). Given the role of Stat 1 as a major signal transducer for type I and type II IFNs, we induced Tregs from naive $\mathrm{CD}^{+}$cells isolated from wild-type B6 mice, Ifnar ${ }^{-1}$ mice, and Ifngr ${ }^{-1}$ mice under Treg conditions to determine the contribution of IFN- $\gamma$ or IFN- $\alpha$ to the generation of iTregs. As shown in Figure 8B, the absence of IFN- $\gamma$ receptor significantly increased Treg generation, whereas the absence of IFN- $\alpha$ receptor had only a modest effect on Treg induction. 

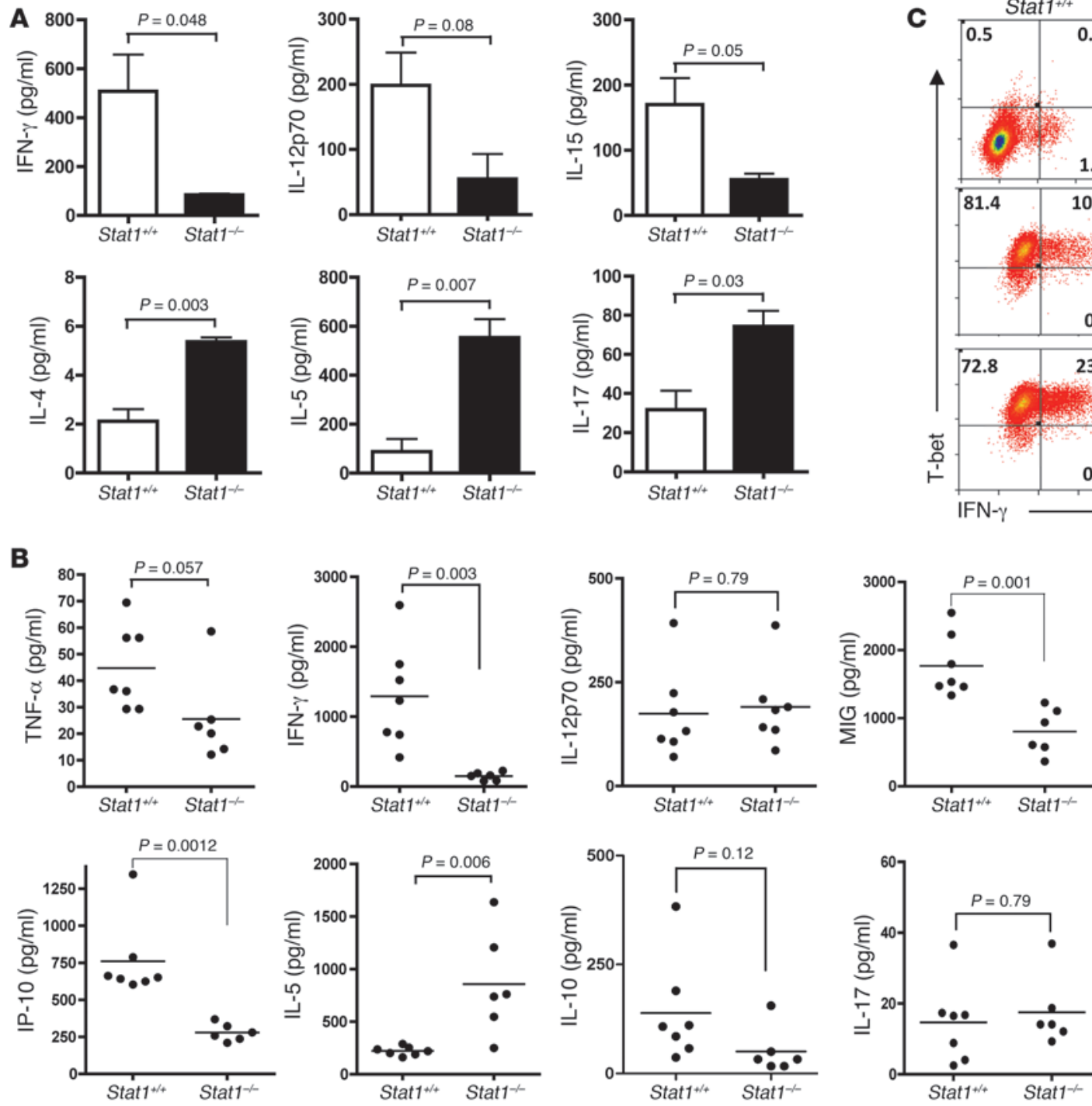

D
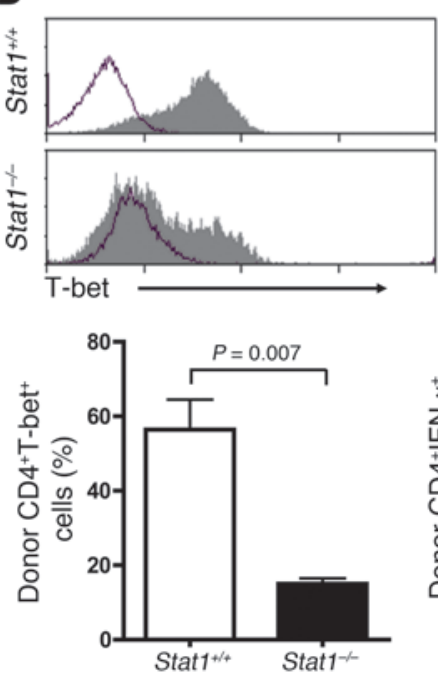

E
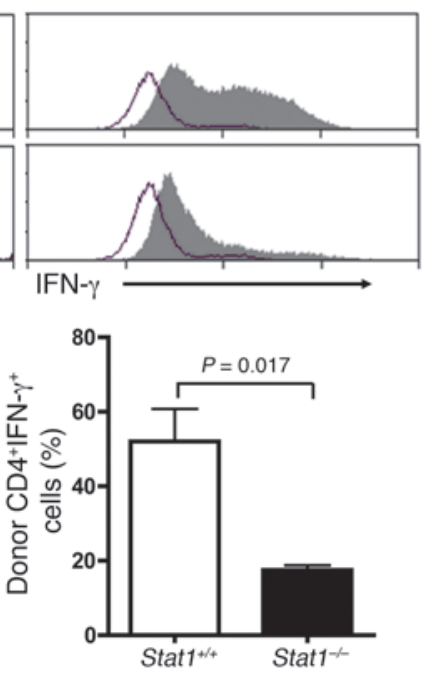

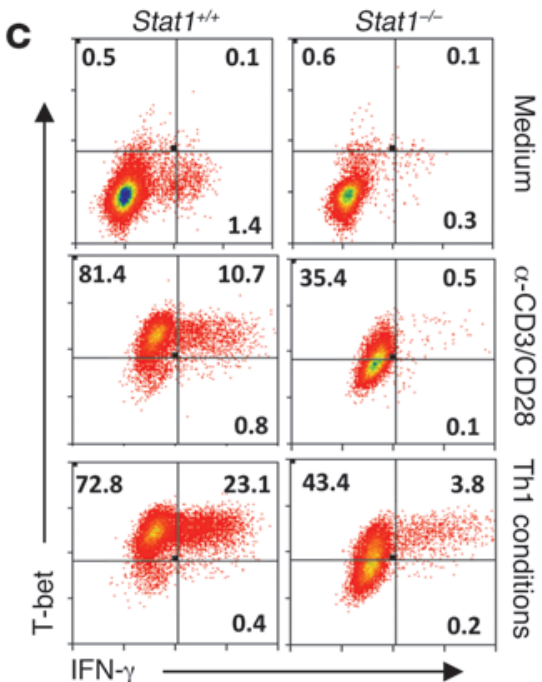

\section{变

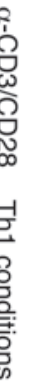




\begin{abstract}
Figure 5
Absence of Stat1 leads to skewed systemic cytokine profile and impairs Th1 differentiation. (A) Serum cytokine profiles were studied on day 6 BMT in MHC-mismatched BMT with 3-4 animals per group. Data are representative of 1 of 3 independent experiments. (B) Serum cytokine profiles on day 6 after BMT following $\mathrm{mHA}$-mismatched BMT with 6-7 animals per group. Serum concentrations of individual animals are shown. Horizontal bars denote mean cytokine serum concentration of the group. (C) Intracellular staining and detection of T-bet and IFN- $\gamma$ in 129. Stat $1^{+/+}$and 129. Stat $1^{-/-}$splenocytes cultured for 3 days on immobilized $\alpha$-CD3 $(5 \mu \mathrm{g} / \mathrm{ml})$ and soluble $\alpha$-CD28 $(1 \mu \mathrm{g} / \mathrm{ml})$ in the absence $(\alpha-C D 3 / C D 28)$ or presence (Th1 conditions) of $\alpha-\mathrm{IL}-4$ antibodies $(10 \mu \mathrm{g} / \mathrm{ml})$ and $10 \mathrm{ng} / \mathrm{ml} \mathrm{IL-12}$. Unstimulated cells cultured in medium alone were used as controls. One representative experiment of 3 independent experiments is shown. Numbers represent the percentages of cells present in each quadrant. (D) Intracellular T-bet and IFN- $\gamma$ expression were determined in donor Stat1 $1^{-/}$and Stat1 ${ }^{+/+}$CD4+ cells following induction of $\mathrm{MHC}$ mismatched GVHD on day 6 . Results of 2 representative animals are shown at top; summary data ( $n=3$ animals/group) are shown in the graphs below. (E) Intracellular IL-4 and IL-17A expression in Stat1+/+ and Stat1 $1^{-/}$CD4 $4^{+}$donor T cells on day 6 following MHC-mismatched BMT and GVHD induction. Histogram bars show mean \pm SEM. Results are representative of 2 separate experiments.
\end{abstract}

Furthermore, adding anti-IFN- $\gamma$ antibodies could increase Treg generation in both wild-type and Ifnar $/-$ cells but did not further enhance Treg induction in the Ifngr ${ }^{-/}$cells. These observations suggest that IFN- $\gamma$ contributes to the regulation Treg generation.

To obtain further supporting evidence for the role of IFN- $\gamma$ induced Stat 1 signaling in the induction of iTreg, Stat $1^{+/+}$ $\mathrm{CD} 4^{+} \mathrm{CD} 25^{-}$cells were cultured under Treg conditions in the absence or presence of anti-IFN- $\gamma$ antibodies for 3 days and studied for $\mathrm{p}$-Stat 1 expression. As shown in Figure 8C, increased p-Stat 1 expression was observed after 3 days of culture under Treg conditions. The addition of $\alpha-\mathrm{IFN}-\gamma$ antibodies almost completely blocked p-Stat 1 expression: as shown in Figure 8D, twice as many $\mathrm{CD} 25^{+} \mathrm{Foxp}^{+}$Tregs were generated from $\mathrm{CD} 4^{+} \mathrm{CD} 25^{-}$ cells during culture in the presence of $\alpha-I F N-\gamma$ antibodies. In addition, induction of iTregs from Stat $1^{-/-} \mathrm{CD} 4^{+} \mathrm{CD} 25^{-}$cells was comparable with iTregs generated from Stat $1^{+/+}$cells in the presence of $\alpha-$ IFN- $\gamma$ antibodies. However, adding $\alpha-I F N-\gamma$ antibodies to Stat $1^{-/-} \mathrm{CD} 4{ }^{+} \mathrm{CD} 25^{-}$cells did not result in a further increase in Tregs, indicating that the inhibitory effects of IFN- $\gamma$ on Treg induction was Stat 1 dependent.

IFN regulatory factor 1 (IRF-1) is a member of the IFN regulatory transcription factor family and is one of the most prominent Stat1-inducible genes in response to IFN- $\gamma(40)$. It was reported that IRF-1 inhibits Treg differentiation by repressing Foxp3 expression through direct binding to a highly conserved IRF consensus element sequence in the promoter of the Foxp3 gene. Furthermore, Irf1-deficient mice had higher numbers of nTregs and iTregs (41). We therefore wanted to know whether the observed increase in Tregs generated from Stat 1 -deficient $\mathrm{CD} 4^{+} \mathrm{CD} 25^{-}$is associated with reduced IRF-1 expression. Using quantitative real-time PCR (qRT-PCR), we demonstrated significantly reduced expression of IRF-1 in Stat1-deficient iTregs. Consistent with this result, the addition of neutralizing $\alpha-$ IFN- $\gamma$ antibodies to Stat 1 wild-type iTreg cultures reduced IRF-1 expression to the low levels observed in Stat1-deficient iTregs (Figure 8E). The addition of $\alpha-$ IFN- $\gamma$ antibodies did not further inhibit IRF-1 expression in
Stat $1^{-/}$iTregs. The qRT-PCR results were confirmed by Western blot (Figure 8F). These findings suggest that the inhibitory effects of IFN- $\gamma /$ Stat 1 on Treg induction may involve IRF-1.

We next sought to confirm enhanced iTreg generation in the absence of Stat 1 using BALB/c mice that were reconstituted with $5 \times 10^{6} \mathrm{TCD} 129$. Stat $1^{+/+} \mathrm{BMCs}$ following lethal irradiation. Recipients were co-injected with $3 \times 10^{6} \mathrm{CD}^{+} \mathrm{CD} 25^{-}$cells purified from either 129.Stat $1^{+/+}$or 129. Stat $1^{-/-}$splenocytes (purity $94 \%)$. BALB/c animals reconstituted with syngeneic BMCs and $\mathrm{CD} 4^{+} \mathrm{CD} 25^{-} \mathrm{T}$ cells served as controls. On day 6 after BMT, animals were sacrificed and spleens were analyzed by flow cytometry (FCM). As expected, we found a significantly higher proportion of $\mathrm{CD} 4^{+} \mathrm{CD} 25^{+}$Foxp $^{+}$cells in recipients of $\mathrm{CD} 4^{+} \mathrm{CD} 25^{-}$Stat $1^{-/-}$cells compared with recipients of Stat $1^{+++}$T cells (Figure 8G).

Stat $1^{-1-}$ Tregs are functional in vitro and in vivo. Next, we assessed whether Stat $1^{-1-}$ Tregs are functional. It has been reported that Foxp $3^{+}$Tregs exert their suppressive effects by a variety of mechanisms, including cell contact-dependent mechanisms and soluble factors, such as TGF- $\beta$ and IL-10. To determine whether Stat $1^{-/-}$ Tregs are different from wild-type Tregs, we first assessed the expression of cytotoxic T lymphocyte antigen-4 (CTLA4) glucocorticoid-induced TNF receptor-related protein (GITR) within the $\mathrm{CD} 4^{+} \mathrm{CD} 25^{+} \mathrm{Foxp}^{+}$population. Although lack of Stat 1 signaling led to an increased proportion of CTLA $4^{+} \mathrm{GITR}^{+}$cells in the donor $\mathrm{CD}^{+}$population on day 6 after BMT, absolute numbers of CTL $\mathrm{A}^{+}{ }^{+} \mathrm{ITR}^{+} \mathrm{CD}^{+} \mathrm{CD}_{25} 5^{+} \mathrm{Foxp}^{+}$cells were not statistically different in recipients of Stat $1^{-/-}$compared to Stat $1^{+/+}$splenocytes (Supplemental Figure 7, A and B). Furthermore, we did not find a significant difference in TGF- $\beta 1$ secretion in supernatants collected from Stat $1^{+/+}$and Stat1 $1^{-/-}$nTreg cultures (Supplemental Figure 7C) or in serum IL-10 levels of Stat $1^{+/+}$versus Stat $1^{-/-}$recipients in both mHA-mismatched and MHC-mismatched BMT settings (Figure $5 \mathrm{~B}$ and data not shown).

We then assessed the suppressive capacity of Stat1-deficient Tregs in vitro. CFSE-labeled 129.Stat $1^{+/+} \mathrm{CD}^{+} \mathrm{CD} 25^{-}$cells were stimulated with $\alpha$-CD3/ $\alpha$-CD28 in the presence of either 129 . Stat $1^{+/+}$or 129. Stat $1^{-/-} \mathrm{CD} 4^{+} \mathrm{CD} 25^{+}$Tregs at a responder/Treg $(\mathrm{R} / \mathrm{T})$ ratio of 10:1 (Figure 9A). Stat1-deficient Tregs suppressed proliferation of TCR-triggered $\mathrm{CD} 4{ }^{+} \mathrm{CD} 25^{-} \mathrm{T}$ cell responder cells even at a low $\mathrm{R} / \mathrm{T}$ ratio (Figure 9A). To exclude the possibility that enhanced proliferation of the Stat $1^{-/}$Tregs leading to a skewed $\mathrm{R} / \mathrm{T}$ ratio during the time of co-culture accounted for the inhibition of the responder population, we also conducted experiments in which Tregs were irradiated with 3,000 cGy. Even under these conditions, we observed a Treg-dependent inhibition of responder cell proliferation (data not shown) by both Stat $1^{+/+}$and Stat $1^{-/-}$ Tregs. Thus, these results suggested that Stat1-deficient Tregs were functional in vitro but did not reveal any major differences regarding the suppressive mechanisms.

Next we attempted to confirm the preserved function of Stat1deficient Tregs in vivo. Tregs have been reported to effectively inhibit the induction of acute GVHD (32). For this purpose, we used Stat $1^{-/-}$or $S t a t 1^{+/+}$Tregs that had been cultured for 3 days in vitro in the presence of $\alpha$-CD3/ $\alpha$-CD28, IL- 2 , and TGF- $\beta 1$. Under these conditions, more than $95 \%$ of $\mathrm{CD}^{+} \mathrm{T}$ cells from both $\mathrm{Stat1}^{+/+}$ and Stat $1^{-/-}$donors retained Foxp $3^{+}$expression (Figure 7D), compared with cultures without TGF- $\beta 1$ (Figure $7 \mathrm{C}$ ). Lethally irradiated $\mathrm{BALB} / \mathrm{C}$ mice were reconstituted with $5 \times 10^{6} \mathrm{TCD} \mathrm{BMC}$, and $5 \times 10^{5}$ pan-T cells from 129 . Stat $1^{+/+}$mice plus $5 \times 10^{5}$ in vitrocultured 129.Stat $1^{+/+}$or 129. Stat $1^{-/-}$Tregs. BALB/c mice receiving 
A

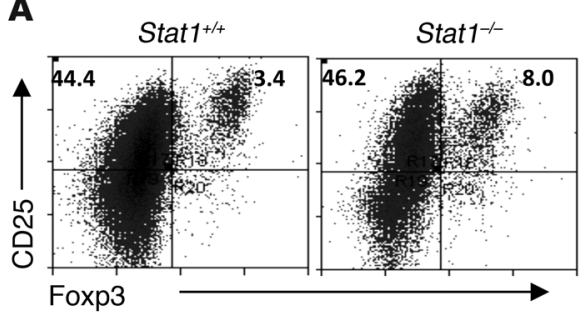

B

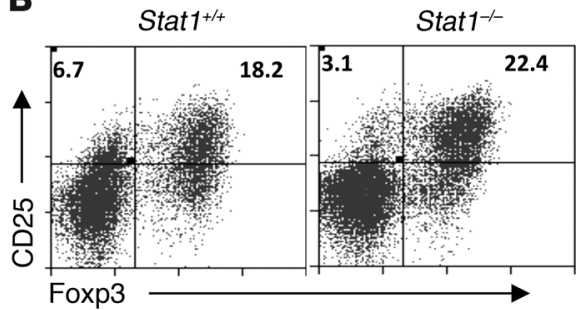

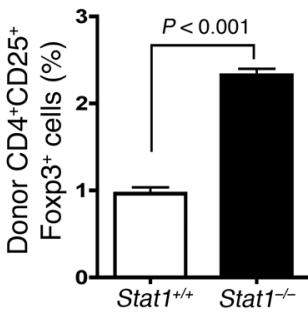
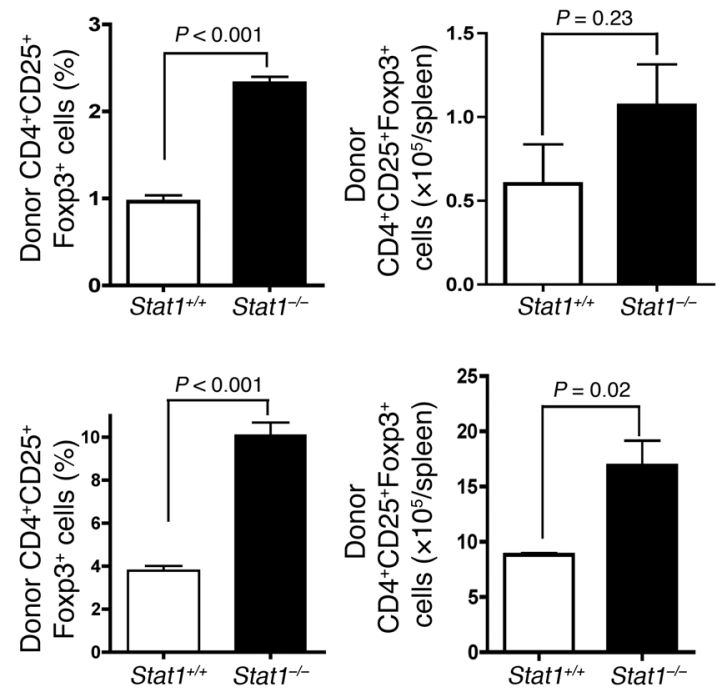

Figure 6

Lack of Stat1 in donor lymphocytes leads to increased Foxp3+ Tregs. (A) Splenocytes were harvested on day 6 following MHC-mismatched BMT and examined for the presence of donor CD4+CD25+Foxp3 ${ }^{+}$Tregs. CD25 and Foxp3 expression were assessed in donor CD4 cells. Foxp3 ${ }^{+}$Tregs increased significantly in MHC-mismatched recipients of Stat1 ${ }^{-/}$versus Stat $1^{+/+}$splenocytes. One representative experiment of 5 independent experiments with 3-5 animals per group. (B) Absence of Stat1 leads to increased Treg expansion in mHA-mismatched BMT. Representative results from 1 of 2 independent experiments are shown ( $n=3 / g r o u p)$. The left panels show dot plots of representative animals receiving Stat $1^{+/+}$or Stat1-/- splenocytes. The right panels show summarized data of the relative proportions and absolute cell numbers of Tregs. Numbers in the dot plots represent the percentages of cells present in the given quadrants.

129. Stat $1^{+/+}$TCD BMCs and pan-T cells only were used as GVHD controls, while lethally irradiated mice receiving TCD BALB/c BMCs and pan- $T$ cells were used as syngeneic controls. As shown in Figure 9B, in vitro-expanded Tregs from both Stat $^{+/+}$and Stat $1^{-/-}$mice led to complete protection from GVHD at a R/T ratio of $1: 1$. Of note, recipients of Stat1-deficient Tregs lost significantly less weight compared with recipients of Stat $1^{+/+}$Tregs (Supplemental Figure 8). Based on the difference in weight loss, we hypothesized that Stat1deficient Tregs may be superior to Stat $1^{+/+}$Tregs in suppressing GVHD due to the enhanced proliferation and survival of Stat1deficient Tregs. To address this question, we compared Stat $1^{-/}$and Stat $1^{+/+}$Tregs at different $\mathrm{R} / \mathrm{T}$ ratios (1:4 and 1:8). At a $\mathrm{R} / \mathrm{T}$ ratio of 1:4, both Stat $_{1}{ }^{+/}$and Stat $1^{-/-}$Tregs were equipotent in preventing GVHD mortality (Figure 9C), although recipients of Stat $1^{-/-}$Tregs again showed improved weight curves (data not shown). However, at a ratio of 1:8, Stat $1^{+/+}$Tregs were significantly less potent in preventing GVHD mortality compared with Stat $1^{-/-}$Tregs (Figure 9C). These data therefore suggest that in vitro expanded Stat $1^{-1}$ nTregs have an increased proliferative capacity, leading to enhanced in vivo suppressive function.

\section{Discussion}

The exact roles of type I and II IFNs in the development of GVHD have yet to be fully understood. Multiple lines of evidence indicate that IFN- $\gamma$, a hallmark of Th1 differentiation, may promote (17) or antagonize $(14,18,30)$ GVHD morbidity and mortality and that these opposing effects may depend to some extent on the intensity of conditioning therapy. As elegantly demonstrated by Welniak et al. (13), lack of IFN- $\gamma$ in donor CD4 T cells resulted in accelerated mortality in MHC class II-mismatched recipients following myeloablative conditioning. In contrast, when sublethal conditioning was used, donor IFN- $\gamma$ deficiency ameliorated
GVHD. The mechanisms underlying these opposing observations remain unclear. In contrast, the contribution of IFN- $\alpha$ to GVHD development has not been established.

Because STAT1 is the primary signaling transducer for type I and II IFNs, we examined the role of this signaling pathway during GVHD using Stat1-deficient donors and found that Stat1dependent signaling promotes Th1 polarization through induction of T-bet and IFN- $\gamma$ expression in donor CD4 T cells following allo-activation during GVHD. In accordance with previous findings by our laboratory $(26,42)$ and other investigators $(43)$, IFN- $\gamma$ induced chemokines and their cognate receptors further amplify the inflammatory response and migration of effector cells to the sites of inflammation. Our data demonstrate that the development of GVHD is delayed or abrogated by donor Stat 1 deficiency in both immunogenetic disparities. Although it has been postulated that donor IFN- $\gamma$ is critical for limiting GVHD through induction of AICD (14) in donor T cells, we observed that the role of Stat 1 in GVHD extends beyond mediating IFN- $\gamma$-induced AICD in effector T cells. The attenuated ability of Stat1-deficient donor cells to induce clinical GVHD and associated tissue damage was associated with reduced activation and expansion of donor T cells as well as impaired Th1 polarization, as indicated by absent or reduced T-bet and IFN- $\gamma$ expression. Furthermore, Stat 1 inhibits expansion of nTregs and generation of iTregs, thereby preventing Treg-mediated abortion of the Th1 response. Due to an enhanced proliferative capacity and resistance to apoptosis, Stat1-deficient Tregs were more potent in suppressing GVHD compared with wild-type Tregs.

Activation of donor lymphocytes triggered by host APCs is considered to be a central event in the early induction of GVHD (44). $T$ cell activation involves multiple, rapidly occurring intracellular signaling events that in turn activate transcription of cytokine 

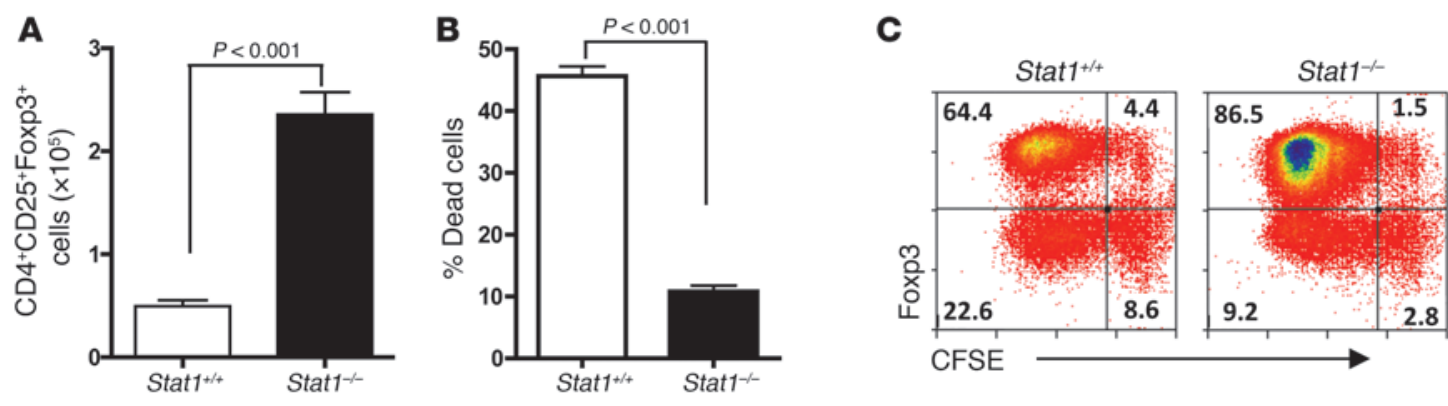

D
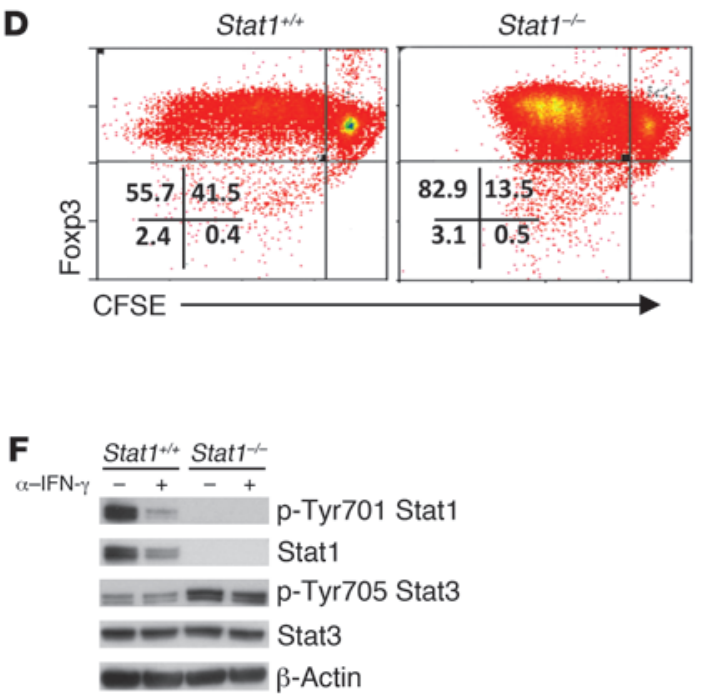

$\mathbf{E}$

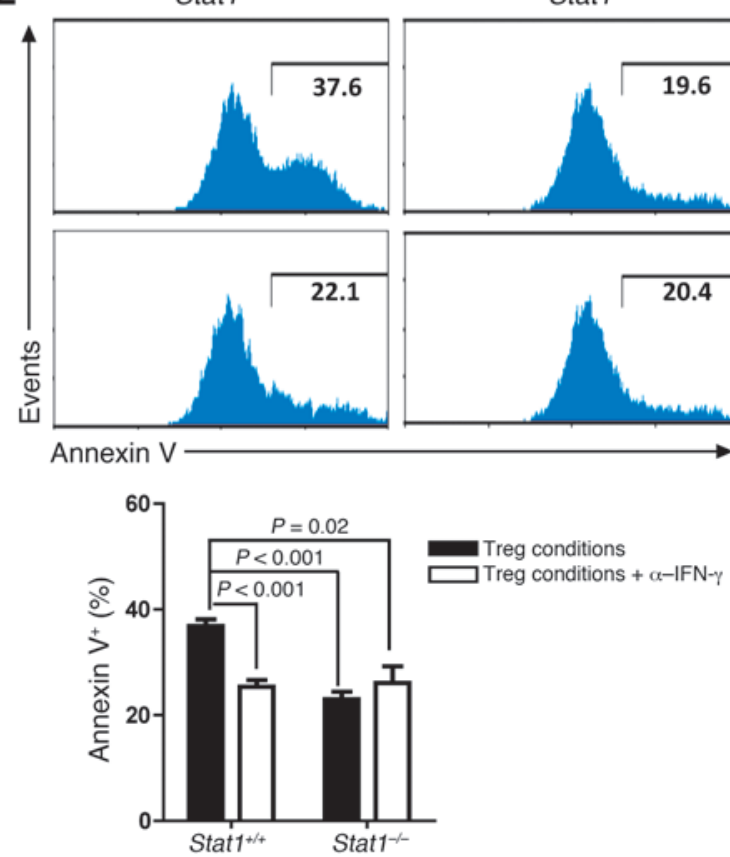

Figure 7

Absence of Stat1 in CD4+ T cells leads to reduced cell death and enhanced proliferation of in vitro-cultured nTregs. (A-D) Freshly purified Stat $1^{+/+}$and Stat $1^{-/-}$CD $4{ }^{+}$CD25 $5^{+}$cells $\left(1 \times 10^{5}\right)$ were labeled with CFSE and stimulated for 3 days with $\alpha-C D 3 / \alpha-C D 28(1 \mu \mathrm{g} / \mathrm{ml})$ antibodies and $\mathrm{IL}-2(10 \mathrm{ng} / \mathrm{ml})$ in the absence (A-C) or presence (D) of $10 \mathrm{ng} / \mathrm{ml}$ human TGF- $\beta 1$. Representative results from 3 independent experiments are shown. (A) Absolute numbers of Foxp3 ${ }^{+}$Tregs were calculated by multiplying viable cell numbers by the percentage of CD25+Foxp3 ${ }^{+}$cells. (B) Viability was determined by Trypan blue staining. Proliferation status of Tregs was analyzed by CFSE dilution in CD4+Foxp $3^{+}$cells in the absence (C) or presence (D) of human TGF- $\beta 1$. Numbers represent the percentages of cells present in the given quadrants. (E and F) Freshly purified Stat $1^{+/+}$and Stat1 ${ }^{-/-}$CD $4+C D 25+$ cells were cultured for 3 days with $\alpha-C D 3 / \alpha-C D 28$ antibodies, IL-2, and TGF- $\beta$ in the absence and presence of $\alpha-\mathrm{IFN}-\gamma(10 \mu \mathrm{g} / \mathrm{ml})$ antibodies. (E) Apoptotic cell death was determined by Annexin $V$ staining using FCM. Top: One representative experiment of 3 independent experiments is shown. Numbers represent the percentages of Annexin V+ cells. Bottom: Bar graph summary of the data. Data are mean \pm SEM. (F) Phosphorylated Tyr701 Stat1 and Tyr705 Stat3 and total Stat1 and Stat3 expression in purified natural Tregs was analyzed by Western blot; $\beta$-actin was used as loading control. Blots are representative of 3 independent experiments.

genes, such as IL-2, IFN- $\gamma$, and their respective receptors $(24,25)$. Whereas Stat 1 deficiency abrogated GVHD in the mHA-mismatched setting, GVHD was only delayed in recipients of fully MHC-mismatched cells, with clear signs of GVHD tissue damage and detection of host-reactive donor T cells (Supplemental Figure 3). In fact, we observed much more vigorous proliferation of donor T cells following MHC-mismatched transplantation (Supplemental Figure 4) compared with the mHA-mismatched setting. Nonetheless, there remained attenuated activation of Stat1-deficient cells (reduction in $\mathrm{CD} 44^{+} \mathrm{CD} 62 \mathrm{~L}^{-} \mathrm{CD} 4{ }^{+} \mathrm{T}$ cells) and decreased expansion of donor T cells (reduced absolute CD4 and CD8 numbers, increased CFSE ${ }^{\text {hi }}$ cells), indicating that reduced activation and proliferation is the main mechanism of reduced GVHD induction by Stat1-deficient T cells.

This reduced activation and proliferation in Stat $1^{-/-}$donor lymphocytes may be counterbalanced by reduced AICD due to the absence of IFN- $\gamma /$ Stat 1 signaling. Data from our laboratory (Supplemental Figure 5) and other laboratories (45) demonstrate hyperproliferation of Stat1-deficient CD4 T cells. Thus, the reduced expansion of Stat1-deficient donor $\mathrm{T}$ cells could not be explained by an intrinsic defect of Stat1-deficient T cells to proliferate. Our results suggest that other mechanisms must explain the reduced expansion of donor $\mathrm{T}$ cells and attenuated GVHD. Indeed, we show that the absence of Stat 1 significantly 
A

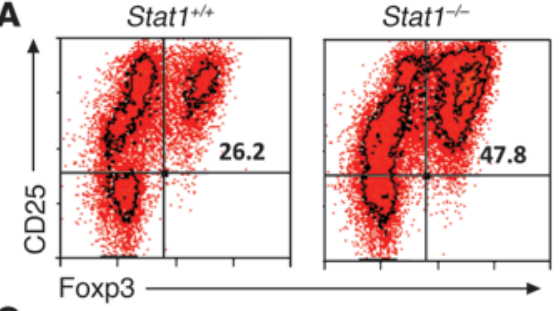

C

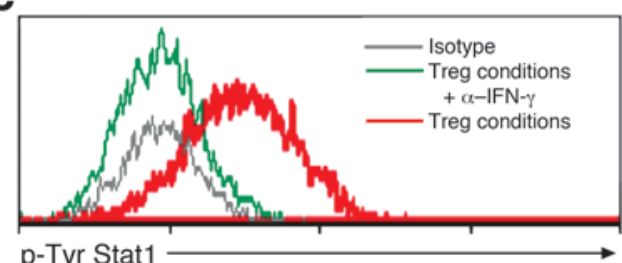

p-Tyr Stat1

D

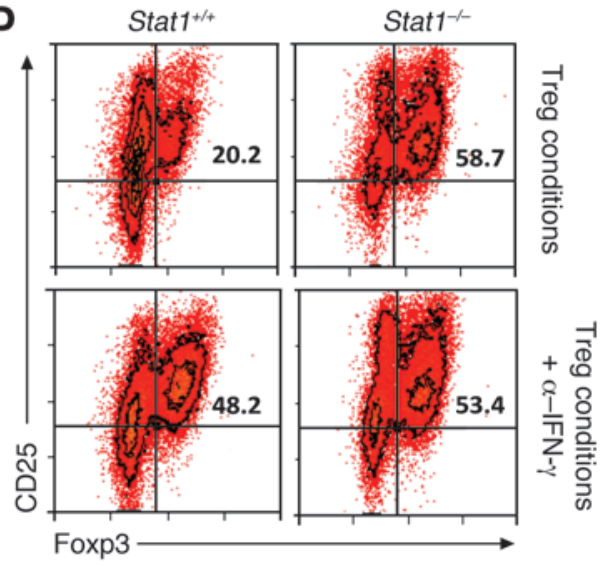

B
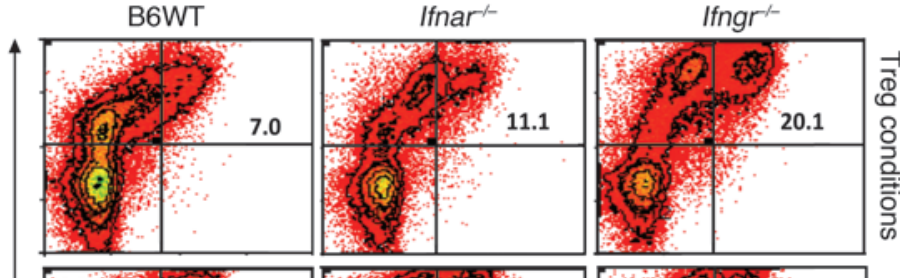

ญึ

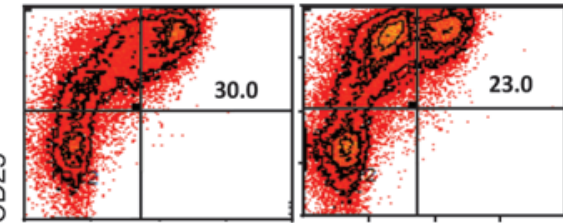

Foxp3

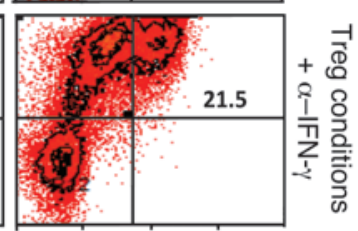

E

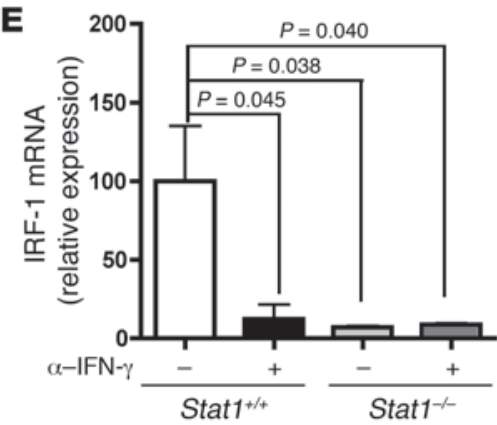

F $\quad \underline{S t a t 1^{+/ \alpha}} \underline{S t a t 1^{-\alpha}}$ $\alpha-I F N-\gamma-++$

p-Tyr701 Stat1

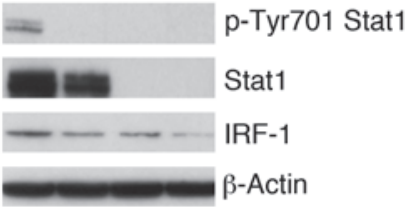

G
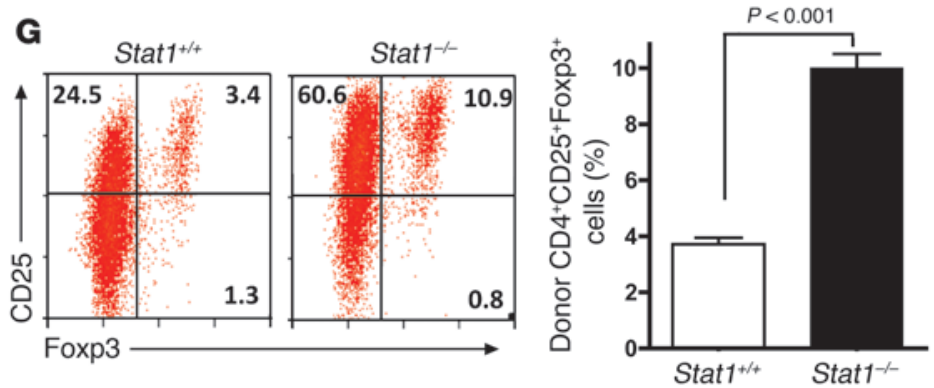

Figure 8

Stat1 deficiency in CD4+ T cells leads to enhanced in vitro and in vivo induction of iTregs in an IFN- $\gamma /$ Stat1-dependent manner. (A) Freshly isolated CD4+CD25- cells from Stat $1^{+/+}$and Stat1 ${ }^{-/-}$mice were cultured for 3 days under Treg conditions to generate iTregs. CD4 ${ }^{+}$CD25 ${ }^{+}$Foxp3 ${ }^{+}$ cells were analyzed by FCM. Numbers represent the percentages of cells in the given quadrant. (B) Freshly isolated naive CD4+T cells from B6 wild-type, B6.Ifnar ${ }^{-1}$, and B6.Ifngr $r^{-1}$ mice were cultured for 3 days under Treg conditions to induce Tregs in the presence or absence of $\alpha-I F N-\gamma$ antibodies. CD4 ${ }^{+} \mathrm{T}$ cells were studied for $\mathrm{CD} 25^{+} \mathrm{Foxp} 3^{+}$expression by FCM. Numbers represent the percentages of cells present in the given quadrants. (C-F) Freshly isolated wild-type CD4 ${ }^{+} \mathrm{CD} 25^{-}$cells were cultured under Treg conditions with or without $\alpha-I F N-\gamma$ antibodies for 3 days. (C) Phosphorylation of Tyr701 Stat1 was detected by FCM. (D) CD4 ${ }^{+}$CD25 ${ }^{+}$Foxp3 ${ }^{+}$cells were assessed. Numbers represent the percentages of cells in the given quadrants. (E) Irf1 mRNA expression was determined by qRT-PCR. (F) Phosphorylation of Tyr701 Stat1 and IRF-1 expression were studied by Western blot analysis, with $\beta$-actin as loading control. (G) iTregs were generated in vivo by transferring $3 \times 10^{6} 129$. Stat $1^{+/+}$or 129. Stat1 ${ }^{-1-}$ CD4+CD25- T cells into lethally irradiated BALB/c mice. Donor-derived CD4+CD25+Foxp3+ iTregs were assessed on day 6 after infusion. Representative results from 3 independent experiments are shown. Numbers represent the percentages of cells in the given quadrants. Data bars show mean \pm SEM.

impaired Th1 differentiation. Furthermore, we show that Stat1 acts as a negative regulator of donor Treg development during the induction of GVHD.

The balance of $\mathrm{p}$-Stat $1 / \mathrm{p}$-Stat 3 is important for the regulation of cell proliferation and apoptosis $(38,39,46)$. We observed that Stat1-deficient purified nTregs displayed increased Stat 3 activation. Therefore, our results might provide a mechanistic explanation for the enhanced proliferation and apoptosis resistance of these cells. In contrast, we observed predominant downregulation of total Stat 1 expression in wild-type nTregs following IFN- $\gamma$ neutralization, without upregulation of p-Stat 3 expression. It is well documented that $\mathrm{p}$-STAT1 homodimers following IFN- $\gamma$ stimulation can bind to the $\gamma$-activating sequence in the promoter region of both STAT1 and IRF1, thus leading to a positive feedback loop promoting STAT1 transcription $(47,48)$. We therefore assume that the blockade of IFN- $\gamma$ results in almost complete abroga- 
A

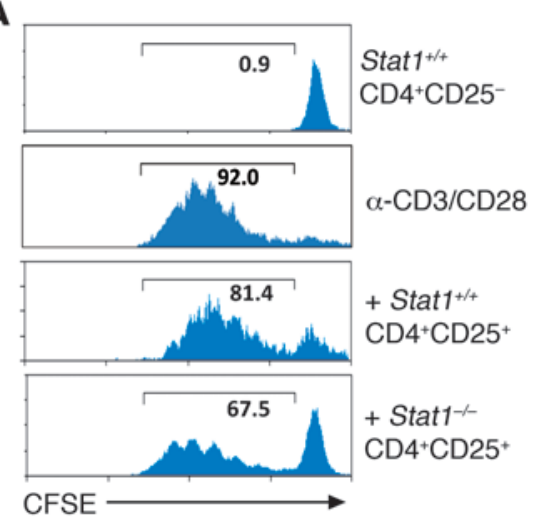

B

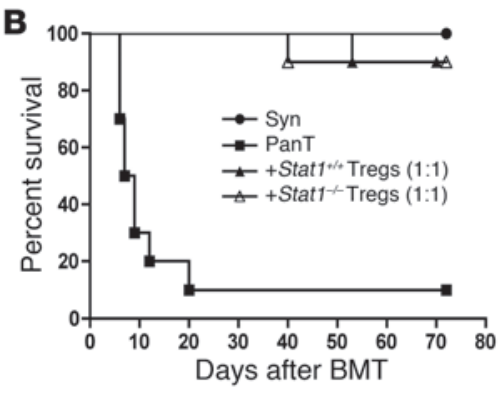

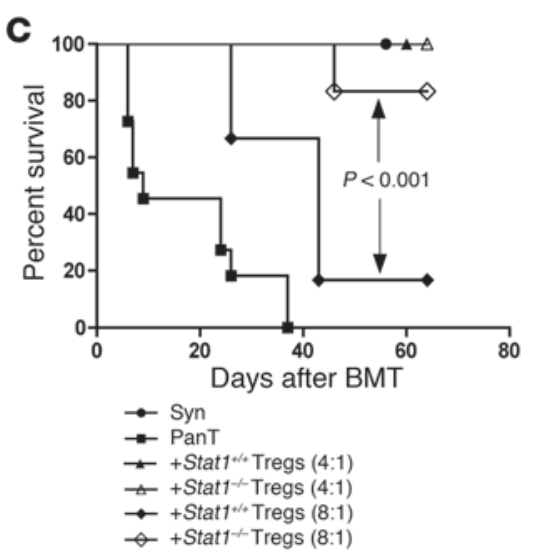

Figure 9

Absence of Stat1 signaling in Tregs does not impair in vitro or in vivo suppressive function. (A) Suppressive function of Tregs isolated from 129. Stat1 ${ }^{-/-}$and 129 . Stat $1^{1 /+}$ mice was tested in vitro by analyzing proliferation of CFSE-labeled Stat $1^{1 /+}$ CD4+CD25- responder T cells stimulated with $\alpha-C D 3 / C D 28$ antibodies in the presence of either Stat1+/+ or Stat1-/- Tregs at a R/T ratio of 10:1. Numbers in the histogram represent the percentages of proliferating CFSElo cells. (B and $\mathbf{C}$ ) In vivo suppressive function of Tregs. Lethally irradiated BALB/c mice were reconstituted with $5 \times 10^{6} \mathrm{BALB} / \mathrm{C}$ TCD BMCs (Syn). For induction of GVHD, $5 \times 10^{6} \mathrm{Stat1+/+}$ TCD BMCs plus $5 \times 10^{5} \mathrm{Stat1}{ }^{+/+}$pan-T cells (PanT) were administered. In vitro-expanded Stat1 ${ }^{+/+}$and Stat1 ${ }^{-/-}$CD4+CD25 $n$ Tregs were added at different R/T ratios. Survival curves are shown for R/T ratios of 1:1 (B) and 4:1 and 8:1 (C). Cumulative data from 2 experiments are shown with 11-12 animals per group.

tion of IFN- $\gamma \mathrm{R}$-dependent Stat 1 activation and subsequent Stat1 expression. Because cells were studied on day 3 of culture, we postulate that Stat 1 expression was already completely downregulated and that IFN- $\gamma$ may promote Stat 1 gene transcription through the MAPK pathway (49). It is conceivable that the complete absence of Stat 1 may lead to constitutive Stat 3 activation in nTregs, promoting their survival and proliferation. In line with these considerations, Stat3 inhibition using siRNA has been shown to inhibit Foxp3 expression and block Tregs (50). In wild-type Tregs, neutralization of IFN- $\gamma$ resulted in a profound downregulation of Stat 1 activation and expression but did not influence the expression levels of activated Stat3. We therefore postulate that although expression of activated $\mathrm{p}$-Stat 3 did not change, the $\mathrm{p}$-Stat $1 / \mathrm{p}$-Stat 3 ratio still favored Stat3 signaling and promoted Stat3-driven survival and proliferation $(38,46)$. Additional studies are warranted to delineate the precise crosstalk of Stat 1 and Stat 3 in Tregs.

Lack of Stat 1 also led to a significant enhancement of iTreg generation. In this context, we demonstrate that Stat1-deficient iTregs have a significantly lower expression of IRF-1, which has been shown to be a negative regulator of Foxp 3 expression and Treg generation (41). We do not provide direct mechanistic evidence that the decrease in IRF-1 expression observed in Stat1-deficient Tregs or following IFN- $\gamma$ blockade directly accounts for the enhanced generation of iTregs. However, it is possible that attenuated IRF-1 expression may be at least one mechanism leading to enhanced iTreg generation. We therefore postulate that under physiologic conditions, the inhibitory effect of Stat 1 on Treg development may contribute to preventing premature Treg-dependent abrogation of a Th1 inflammatory response. Taken together, our findings provide evidence that the absence of Stat 1 promotes the generation and expansion of Tregs and that this Stat1-dependent suppression of Tregs is primarily due to IFN- $\gamma$, and not IFN- $\alpha$, signaling.

Most importantly, we show that in Stat $1^{-/}$Tregs, the functional markers (Foxp3, CTLA4, GITR, TGF- $\beta$, and IL-10) were fundamentally comparable with their Stat $1^{+/+}$counterparts. Consistent with our observation that Stat $1^{-/-}$Tregs have an enhanced pro- liferative capacity and resistance to apoptosis, we demonstrated that ex vivo-expanded Stat1-deficient nTregs were significantly more potent than wild-type Tregs in suppressing GVHD. Based on these results, we postulate that the enhanced proliferative capacity, together with the increased resistance to apoptosis, is the primary reason for the superiority of Stat1-deficient Tregs compared with wild-type Tregs.

Currently, the role of Stat 1 in the development of Tregs is controversial due to conflicting results. Nishibori et al. noted that Stat1-deficient animals are more susceptible to the development of experimental autoimmune encephalitis (EAE) due to impaired development and function of Tregs (34). In contrast, using this same model with enhanced susceptibility to EAE induction, Bettelli et al. were not able to confirm a functional Treg defect in Stat1-deficient mice (51). In support of our findings, recent studies suggest that IFN- $\gamma$-dependent signaling and Th1 conditions may be negative regulators of Treg development $(52,53)$.

In contrast to the mHA-mismatched model, Stat1 deficiency merely delayed GVHD-induced mortality in a fully MHC-mismatched setting. We observed that Stat 1 deficiency in $\mathrm{CD}^{+} \mathrm{T}$ cells does not impair their in vitro differentiation into IFN- $\gamma$-producing cells if exogenous IL-12 is present, nor their in vivo differentiation during induction of GVHD (H. Ma and M.Y. Mapara, unpublished observations). Thus, it is possible that donor Stat $1^{-/-} \mathrm{CD} 8 \mathrm{~T}$ cells might also potentially contribute to the development of delayed GVHD in the MHC-mismatched setting.

More importantly, we noted an increased Th17 differentiation in the MHC-mismatched setting, which was not observable in the mHA-mismatched setting. The role Th17 in experimental GVHD has led to seemingly discordant results. Some reports suggest that Th17 cells promote induction of GVHD $(54,55)$, while others report that Th17 differentiation attenuates the induction of acute GVHD by diminishing the Th1 response (56). It is therefore possible that this Th17 skewing of Stat1-deficient donor cells may account for the delayed mortality in the MHC-mismatched model. In addition, our data suggest that Th17 differentiation may be 
influenced by the extent of the immunogenetic disparity and the intensity of the allostimulation. Further studies are currently ongoing to clarify the contribution of Th17 cells to the delayed GVHD seen in our MHC-mismatched model.

Our finding that lack of Stat 1 reduces GVHD is at odds with the notion that IFN- $\gamma$-dependent induction of AICD is a limiting factor for the development of GVHD. Several non-mutually exclusive explanations might account for this apparent contradiction. First, the absence of Stat 1 leads to reduced activation and impaired Th1 differentiation, as shown in this study, while IFN- $\gamma$ deficiency did not attenuate activation, as reported by others $(11,30)$. Secondly, based on our results, we postulate that IFN- $\gamma$ will not only induce AICD in donor effector T cells but will also suppress the development of Tregs. Therefore, in the absence of IFN- $\gamma /$ Stat 1 -dependent signaling, it may be that the balance of increased Treg expansion rather than reduced AICD of the effector T cells influences the development of GVHD. Given the significantly reduced GVHD in both BMT settings, it appears that the increased expansion of Tregs as well as the attenuated Th1 differentiation trumped the reduced AICD. Furthermore, it is possible that IFN- $\gamma$-mediated suppression of Tregs is critically dependent on the presence of Stat 1 , whereas IFN- $\gamma$-dependent AICD of donor T cells may utilize Stat1-independent signaling pathways (57). In addition, the role of IFN- $\gamma$ in regulating GVHD is influenced by conditioning intensity (13) and may further depend on the presence and intensity of allostimulation from host hematopoietic cells.

Thus, it is not possible to equate IFN- $\gamma$ deficiency with STAT1 deficiency. IFN- $\gamma$ has been shown to be important for mediating the lymphohematopoietic graft-versus-host reaction (18), and lack of donor IFN- $\gamma$ may result in reduced and delayed clearance of host APCs, leading to prolonged and more intense allostimulation of the donor $\mathrm{T}$ cells, which in turn leads to massive expansion and reduced contraction due to impaired AICD.

In addition, the absence of STAT1 is associated with several defects in cellular immunity, resulting in an enhanced susceptibility to viral, Mycobacterium tuberculosis, and Leishmania major infections (58-62). The mechanistic basis for this immunodeficiency involves impaired memory development, CTL generation, and DC function (63-65). Our results now provide evidence that Stat 1 is an inhibitor of Tregs and that in the absence of Stat1, enhanced expansion of Tregs may lead to blunting of the Th1 response, thereby contributing to immunosuppression. However, despite these immune defects, our model demonstrated that Stat1-deficient donors were still able to launch anti-host responses in the fully MHC-mismatched setting. In addition, we saw retained anti-third party responses in $S t a t 1^{-/-}$BALB/c chimeras (data not shown). Finally, we have evidence that CD8 T cells are not impaired in their cytolytic activity (H. Ma and M.Y. Mapara, unpublished observations). Taken together, these findings indicate that, despite the well-established immune defects induced by Stat 1 deficiency, the inhibition of GVHD in our model cannot be attributed to global hyporesponsiveness.

In summary, our studies using Stat1-deficient donors demonstrate how Stat 1 regulates the development of GVHD. First, Stat1deficient CD4 T cells are impaired in their ability to differentiate into Th1 cells, leading to a significant blunting of the inflammatory reaction. Second, absence of Stat 1 in splenocytes is associated with reduced $\mathrm{T}$ cell activation and alloantigen-driven expansion of donor T cells. Third and most significantly, Stat 1 is an inhibitor to both nTreg and iTregs in vitro and in vivo. These latter find- ings have clear implications beyond the realm of GVHD. Based on our observation of enhanced in vitro expansion of nTregs and enhanced generation of iTregs from $\mathrm{CD} 4^{+} \mathrm{CD} 25^{-} \mathrm{T}$ cells, additional studies are warranted, given that targeting Stat 1 may be a promising approach for enhancing in vitro and in vivo generation of Tregs for therapeutic purposes in the context of transplantation or inflammatory conditions.

\section{Methods}

Mice. Female C57BL/6 (B6; H2 $\left.{ }^{\mathrm{b}}\right)$, congenic B6.SJL $\left(\mathrm{H} 2^{\mathrm{b}}\right)$, BALB/c $\left(\mathrm{H} 2^{\mathrm{d}}\right)$, wild-type 129S6/SvEv (129.Stat1 $\left.{ }^{+/+} ; \mathrm{H}_{2}^{\mathrm{b}}\right)$, and 129.Stat1 $1^{-/}$mice were purchased from The Jackson Laboratory or Taconic. Splenocytes from Ifngr ${ }^{-1}$ mice on a B6 background were provided by Kyle C. McKenna (University of Pittsburgh Medical Center, Pittsburgh, Pennsylvania, USA). Ifnar- mice on a 6 background were originally provided by Murali-Krishna Kaja (University of Washington, Seattle, Washington, USA), and we have since maintained breeding colonies (66). All mice were used at 8-12 weeks of age and were housed in autoclaved microisolator environments and provided with sterile water and irradiated food ad libitum. All manipulations were performed in a laminar flow hood. All animal procedures were approved by the IACUC of the University of Pittsburgh.

$B M T$ and induction of GVHD. GVHD was induced in the fully MHC-mismatched strain combination 129.Stat $1^{+/} / 129$. Stat $1^{-/-} \rightarrow \mathrm{BALB} / \mathrm{c}$ or the mHA-mismatched 129. Stat $1^{+/+} / 129$. Stat $1^{-/-} \rightarrow \mathrm{B} 6$ combination. Recipients were lethally irradiated (BALB/c: 800 cGy; B6 and B6.SJL: 1,075 cGy) and reconstituted with a single intravenous inoculum of TCD $5 \times 10^{6}$ allogeneic or syngeneic BMCs. T cell depletion was performed using CD90.2 microbeads (Miltenyi Biotec). In the MHC-mismatched and MHC-matched mHA-mismatched strain setting, GVHD was induced by co-injection of allogeneic spleen cells or selected $\mathrm{T}$ cell populations as described below. Mice from different treatment groups were randomly assigned to different cages to ascertain that caged housed animals from different groups to rule out cage-related effects.

Clinical GVHD assessment and histopathology scoring. GVHD morbidity was assessed using a standard scoring system, which is based on summation of 5 criteria scores: percent of weight change, posture, activity, fur texture, and skin integrity (67). For histopathological analysis of GVHD target tissues, samples were collected from liver and small and large intestines and fixed in $7 \%$ formalin. Formalin-preserved tissue samples were embedded in paraffin, cut into $5-\mu \mathrm{m}$-thick sections, and stained with hematoxylin and eosin for histological examination. A semiquantitative scoring system was used to assess the morphologic changes indicative of GVHD as published by Hill et al. (67): small intestine - villous blunting, crypt regeneration, luminal sloughing of cellular debris, crypt cell apoptosis, crypt destruction, lamina propria inflammatory infiltrate, and mucosal ulceration; colon - crypt regeneration, surface colonocyte vacuolization, surface colonocyte attenuation, crypt cell apoptosis, crypt destruction, lamina propria inflammatory infiltrate, and mucosal ulceration; and liver - portal tract expansion by chronic inflammation, lymphocytic infiltrate of bile duct epithelial apoptosis, bile duct epithelial cell sloughing, endothelialitis, parenchymal apoptosis, parenchymal microabscesses, parenchymal mitotic figures, hepatocellular cholestasis, and steatosis. The scoring system denoted 0 as normal, 0.5 as focal and rare, 1.0 as focal and mild, 2.0 as diffuse and mild, 3.0 as diffuse and moderate, and 4.0 as diffuse and severe. Scores were added to provide a total score for each specimen. Slides were coded without reference to mouse strain or treatment and were systematically examined.

Cytokine multiplex analysis. The LabMap serum/supernatant assays were performed in a 96-well microplate format according to a protocol provided by the manufacturer (BioSource International). A filter-bottom, 96-well microplate (Millipore) was blocked for 10 minutes with PBS/BSA. To gen- 
erate a standard curve, 5-fold dilutions of appropriate standards were prepared in supernatant culture medium. Serum samples were analyzed using the mouse cytokine/chemokine Lincoplex assay (Lincoresearch). Analysis of experimental data was performed using a 5-parametric-curve fitting.

Cell selection procedures. Pan T cells were selected from spleen cells by negative selection (no-touch preparation) using the Pan T Isolation Kit (Miltenyi Biotec) according to the manufacturer's recommendation. Splenocytes were enriched for naive $\mathrm{CD} 4^{+} \mathrm{T}$ cells by negative selection using the $\mathrm{CD} 4^{+}$ Isolation Kit (Miltenyi Biotec) according to the manufacturer's procedure. A purity of at least $92 \%$ was achieved as determined by $\mathrm{FCM}$. CD $4^{+} \mathrm{CD} 25^{+}$ Tregs were purified from splenocytes using the $\mathrm{CD} 4^{+} \mathrm{CD} 25^{+}$Regulatory $\mathrm{T}$ Cell Isolation Kit (Miltenyi Biotec). All procedures were performed according to the manufacturer's recommendations. T cells were labeled with $5 \mu \mathrm{M}$ CFSE (Invitrogen) to determine in vitro and in vivo proliferation according to standard procedures $(29,68)$.

In vitro Th1 and Treg conditions. Splenocytes from Stat $1^{+/+}$or Stat $1^{-/-}$mice were stimulated with plate-bound $\alpha$-CD3 $(1-5 \mu \mathrm{g} / \mathrm{ml}$; clone $145-2 \mathrm{C} 11)$ and soluble $\alpha$-CD28 ( $1 \mu \mathrm{g} / \mathrm{ml}$; clone 37.51 ) (referred to hereafter as $\alpha$-CD3/CD28 thereafter) in 24-well plates (Corning Life Science). For Th1 differentiation, splenocytes were cultured with murine IL-12 (10 ng/ml; Peprotech) plus $\alpha-I L-4$ $(10 \mu \mathrm{g} / \mathrm{ml}$; clone BVD4-1D11) for 72 hours (Th1 conditions). iTregs were generated by culturing purified $\mathrm{CD} 4^{+} \mathrm{CD} 25^{-}$cells in the presence of human TGF- $\beta 1(10 \mathrm{ng} / \mathrm{ml})$ and murine IL-2 $(20 \mathrm{ng} / \mathrm{ml})$ in addition to CD3/CD28 stimulation for 72 hours (Treg conditions). Phenotypes of all differentiated cells were confirmed by FCM. All antibodies were from BD Biosciences.

In vitro and in vivo functional analysis of $\mathrm{CD} 4^{+} \mathrm{CD} 25^{+}$Tregs. $\mathrm{CD} 4^{+} \mathrm{CD} 25^{+}$Tregs were purified from splenocytes of 129 . Stat $1^{+/+}$or 129 .Stat $1^{-/-}$mice using the $\mathrm{CD} 4{ }^{+} \mathrm{CD} 25^{+}$Regulatory $\mathrm{T}$ Cell Isolation Kit (MACS), achieving a purity of more than $95 \%$ based on Foxp3 expression. To assess their proliferative capacity, purified Tregs were labeled with CFSE and cultured under $\alpha$-CD3/CD28 conditions plus murine IL-2 (10 ng/ml; Peprotech) in complete culture medium (RPMI-1640 medium [Lonza]) supplemented with $10 \%$ fetal bovine serum (Hyclone), L-glutamine $2 \mathrm{mM}, 100 \mathrm{U} / \mathrm{ml}$ penicillin, $100 \mu \mathrm{g} / \mathrm{ml}$ streptomycin, and $5 \times 10^{-5} \mathrm{M}$ 2-ME (Sigma-Aldrich) for 72 hours. The expanded Tregs were then analyzed by FCM. The suppressive function of Tregs was assessed by coculturing CFSE-labeled Stat $1^{+/+}$ $\mathrm{CD} 4^{+} \mathrm{CD} 25^{-}$responder cells with Stat $1^{+/+}$or Stat $1^{-/-}$Tregs (irradiated with $3,000 \mathrm{cGy}$ ) at different $\mathrm{R} / \mathrm{T}$ ratios under $\alpha$-CD3/CD28 conditions. The proliferation of CFSE-labeled responder cells was assessed by FCM after 3 days of culture. For GVHD studies, $\mathrm{Stat1}^{+/+}$or Stat1 $1^{-/} \mathrm{CD} 4^{+} \mathrm{CD} 25^{+}$cells were expanded in vitro under Treg conditions and intravenously injected at different $\mathrm{R} / \mathrm{T}$ ratios.

Flow cytometric analysis. Spleens were harvested and gently teased in ammonium chloride potassium (ACK) lysing buffer (BioWhittaker). Single-cell suspensions were filtered through nylon mesh. Splenocytes were prepared and analyzed using FCM with a Beckman Coulter CyAN 9-color High Speed Flowcytometer and Summit 4.3 software (DakoCytomation). The following antibodies were used for these studies: rat $\alpha$-mouse CD4 (H129.19 or RM4-5), rat $\alpha$-mouse CD8a (53-6.7), rat $\alpha$-mouse IFN- $\gamma$ (XMG1.2), rat $\alpha$-mouse CD62L (MEL-14), hamster $\alpha$-mouse CD69 (H1.2F3), rat $\alpha$-CD11b (M1/70), rat $\alpha$-mouse GITR (DTA-1), rat $\alpha$-mouse CD45.2 (Clone 104), mouse $\alpha$-mouse H-2K $\mathrm{K}^{\mathrm{b}}$ (AF6-88.5), mouse $\alpha$-mouse $\mathrm{H}-2 \mathrm{D}^{\mathrm{d}}$ (34-2-12), hamster $\alpha$-mouse CTLA4 (UC10-4F10-11), rat IgG2a (R35-95), hamster IgG1 (G235-2356), mouse IgG2a (G155-178), rat $\alpha-$ IFN- $\gamma$ (XMG1), rat $\alpha$-mouse IL-17A (TC11-18H10), Annexin V, Alexa Fluor 488conjugated anti-Stat1 (pY701) (4a), and Streptavidin APC-conjugated Cy7 purchased from BD Biosciences; and rat $\alpha$-mouse/rat Foxp3 (FJK-16s), rat $\alpha$-mouse CD25 (PC61.5), rat $\alpha$-mouse T-bet (4B10), and Foxp3 Staining Buffer Set purchased from eBioscience. For cell surface staining, isolated cells were stained with the appropriate antibodies for 30 minutes at $4^{\circ} \mathrm{C}$.
For intracellular staining, $1 \times 10^{6}$ isolated cells were stimulated for 4 hours at $37^{\circ} \mathrm{C}$ and $5 \% \mathrm{CO}_{2}$ with PMA $(50 \mathrm{ng} / \mathrm{ml})$ and ionomycin $(1 \mu \mathrm{g} / \mathrm{ml})$ in the presence of monensin $(10 \mu \mathrm{g} / \mathrm{ml})$ in $1 \mathrm{ml}$ complete medium. The cells were then washed, stained with surface antibodies for 30 minutes at $4^{\circ} \mathrm{C}$, fixed/permeabilized, and finally stained for 30 minutes with anti-IFN- $\gamma$, anti-Foxp3, and/or anti-T-bet and anti-CTLA4. For p-Stat1 staining, iTregs were fixed with prewarmed BD Phosflow Lyse/Fix buffer, permeabilized with BD Phosflow Perm Buffer III, and stained with PE-conjugated CD4 (RM4-5) and Alexa Fluor 488-conjugated anti-Stat1 (pY701).

Real-time PCR analysis. For the determination of mRNA levels of IRF-1, total RNA was isolated using Trizol (Invitrogen). Total RNA was converted into cDNA using the Superscript III RT (Invitrogen). Real-time PCR was performed on an ABI Prism 7700 Sequence Detection System (Applied Biosystems). PCR was carried out with SYBR Green PCR Master Mix (Applied Biosystems). The following primers sets were used: IRF-1 sense $5^{\prime}$-ATGCCAATCACTCGAATGCGGA-3', antisense 5'-GGCTGCCACTCAGACTGTTCAA-3'; $\beta$-actin sense 5 '-GAAATCGTGCGTGACATCAAAG-3', and antisense $5^{\prime}$-TGTAGTTTCATGGATGCCACAG-3'.

Western blotting. Extraction of proteins from cultured cells for immunoblotting was performed as previously described using a modified RIPA buffer (69). Total protein lysates $(25-40 \mu \mathrm{g} / \mathrm{ml}$ per lane) were separated by $4 \%-12 \%$ polyacrylamide gel (Bio-Rad). After transfer, blots were incubated with antibodies against p-Stat1 (Tyr701), total Stat1, p-Stat3 (Tyr705), Stat3, IRF-1 (Cell Signaling), and $\beta$-actin (Sigma-Aldrich). Western blots were visualized using enhanced chemiluminescence (Pierce Biotechnology).

In vitro T cell proliferation assay. Bone marrow-derived DCs were generated from BALB/c mice in medium containing murine GM-CSF $(20 \mathrm{ng} / \mathrm{ml}$; Peprotech). On days 6-7, nonadherent cells were harvested and CD11 $c^{+}$ DCs were selected with anti-CD11C $C^{+}$beads (purity $>90 \%$; Miltenyi Biotec) and matured with LPS $(100 \mathrm{ng} / \mathrm{ml})$ for 48 hours. The irradiated cells $(3,000 \mathrm{cGy})$ were cocultured with spleen cells or $\mathrm{CD} 4^{+} \mathrm{T}$ cells from 129.Stat $1^{+/+}$or Stat $1^{-/-}$at different responder/simulator ratios from 1/200 to $1 / 6.25$. After 3 days of culture, cells were pulsed with ${ }^{3} \mathrm{H}$-thymidine $(1 \mu \mathrm{Ci} /$ well $[0.037 \mathrm{MBq}])$ during the last 18 hours of culture and then harvested and counted using the Topcount Microplate (Packard). DNA synthesis was measured using ${ }^{3} \mathrm{H}$-thymidine $\left({ }^{3} \mathrm{H}-\mathrm{TdR}\right)$ incorporation in cpm. All experiments were performed in hexaplicate.

Statistics. Survival data are presented as Kaplan-Meier survival curves, and differences between groups were analyzed using the log-rank test with GraphPad Prism version 5.0 (GraphPad Software). Differences between group means were tested using the 2-tailed Student's $t$ test or the MannWhitney test for nonparametric data. A $P$ value of less than 0.05 was considered to be significant. Data are shown as mean \pm SEM.

\section{Acknowledgments}

This work was supported by NIH grant R01HL093716 and the Pittsburgh Foundation (grant 2007-M0028). M.Y. Mapara is the recipient of the Hillman Fellow Award in Innovative Cancer Research. We would like to thank Hassane Zarour, Angus Thomson, Michael T. Lotze, Richard Steinman, and Michelle Kienholz for critical reading of the manuscript.

Received for publication May 14, 2010, and accepted in revised form April 25, 2011.

Address correspondence to: Markus Y. Mapara, University of Pittsburgh Cancer Institute, Division of Hematology-Oncology, Hillman Cancer Center Research Pavilion, Office Suite 1.19b, 5117 Centre Avenue, Pittsburgh, Pennsylvania 15213-1863, USA. Phone: 412.623.1112; Fax: 412.623.1415; E-mail: maparamy@upmc.edu. 
1. Hsieh MM, et al. Allogeneic hematopoietic stemcell transplantation for sickle cell disease. $N$ Engl J Med. 2009;361(24):2309-2317.

2. Wynn RF, Boelens JJ. Bone-marrow transplantation in non-malignant disease. Lancet. 2009; 374(9693):856-858.

3. Krishnamurti L, et al. Stable long-term donor engraftment following reduced-intensity hematopoietic cell transplantation for sickle cell disease. Biol Blood Marrow Transplant. 2008;14(11):1270-1278.

4. Smith AR, Baker KS, Defor TE, Verneris MR, Wagner JE, Macmillan ML. Hematopoietic cell transplantation for children with acute lymphoblastic leukemia in second complete remission: similar outcomes in recipients of unrelated marrow and umbilical cord blood versus marrow from HLA matched sibling donors. Biol Blood Marrow Transplant. 2009;15(9):1086-1093.

5. Ringden O, Karlsson H, Olsson R, Omazic B, Uhlin M. The allogeneic graft-versus-cancer effect. $\mathrm{BrJ}$ Haematol. 2009;147(5):614-633.

6. Shlomchik WD. Graft-versus-host disease. Nat Rev Immunol. 2007;7(5):340-352.

7. Chakraverty R, Sykes M. The role of antigen-presenting cells in triggering graft-versus-host disease and graft-versus-leukemia. Blood. 2007;110(1):9-17.

8. Welniak LA, Blazar BR, Murphy WJ. Immunobiology of allogeneic hematopoietic stem cell transplantation. Annu Rev Immunol. 2007;25:139-170.

9. Yi T, et al. Reciprocal differentiation and tissue-specific pathogenesis of Th1, Th2, and Th17 cells in graftversus-host disease. Blood. 2009;114(14):3101-3112.

10. Hu X, Ivashkiv LB. Cross-regulation of signaling pathways by interferon-gamma: implications for immune responses and autoimmune diseases. Immunity. 2009;31(4):539-550.

11. Lu Y, Waller EK. Dichotomous role of interferongamma in allogeneic bone marrow transplant. Biol Blood Marrow Transplant. 2009;15(11):1347-1353.

12. Murphy WJ, et al. Differential effects of the absence of interferon-gamma and IL-4 in acute graft-versushost disease after allogeneic bone marrow transplantation in mice. J Clin Invest. 1998;102(9):1742-1748.

13. Welniak LA, Blazar BR, Anver MR, Wiltrout RH, Murphy WJ. Opposing roles of interferon-gamma on CD4+ T cell-mediated graft-versus-host disease: effects of conditioning. Biol Blood Marrow Transplant. 2000;6(6):604-612.

14. Asavaroengchai W, et al. An essential role for IFNgamma in regulation of alloreactive CD8 T cells following allogeneic hematopoietic cell transplantation. Biol Blood Marrow Transplant. 2007;13(1):46-55.

15. Yang YG, Qi J, Wang MG, Sykes M. Donor-derived interferon gamma separates graft-versus-leukemia effects and graft-versus-host disease induced by donor CD8 T cells. Blood. 2002;99(11):4207-4215.

16. Mowat AM. Antibodies to IFN-gamma prevent immunologically mediated intestinal damage in murine graft-versus-host reaction. Immunology. 1989; 68(1):18-23.

17. Burman AC, et al. IFNgamma differentially controls the development of idiopathic pneumonia syndrome and GVHD of the gastrointestinal tract. Blood. 2007;110(3):1064-1072.

18. Wang H, et al. Paradoxical effects of IFN-gamma in graft-versus-host disease reflect promotion of lymphohematopoietic graft-versus-host reactions and inhibition of epithelial tissue injury. Blood. 2009; 113(15):3612-3619.

19. Cleveland MG, Lane RG, Klimpel GR. Enhanced interferon-alpha/beta (IFN-alpha/beta) and defective IFN-gamma production in chronic graft versus host disease: a potential mechanism for immunosuppression. Cell Immunol. 1987;110(1):120-130.

20. Pavord S, Sivakumaran M, Durrant S, Chapman C. The role of alpha interferon in the pathogenesis of GVHD. Bone Marrow Transplant. 1992;10(5):477.

21. Reyes VE, Klimpel GR. Interferon alpha/beta syn- thesis during acute graft-versus-host disease. Transplantation. 1987;43(3):412-416.

22. Gautier G, et al. A type I interferon autocrine-paracrine loop is involved in Toll-like receptor-induced interleukin-12p70 secretion by dendritic cells. J Exp Med. 2005;201(9):1435-1446.

23. Schindler C, Plumlee C. Inteferons pen the JAK-STAT pathway. Semin Cell Dev Biol. 2008;19(4):311-318.

24. Afkarian M, et al. T-bet is a STAT1-induced regulator of IL-12R expression in naive CD4+ T cells. Nat Immunol. 2002;3(6):549-557.

25. Schulz EG, Mariani L, Radbruch A, Hofer T. Sequential polarization and imprinting of type 1 $\mathrm{T}$ helper lymphocytes by interferon-gamma and interleukin-12. Immunity. 2009;30(5):673-683.

26. Leng C, et al. Reduction of graft-versus-host disease by histone deacetylase inhibitor suberonylanilide hydroxamic acid is associated with modulation of inflammatory cytokine milieu and involves inhibition of STAT1. Exp Hematol. 2006;34(6):776-787.

27. Reddy P, Negrin R, Hill GR. Mouse models of bone marrow transplantation. Biol Blood Marrow Transplant. 2008;14(1 suppl 1):129-135.

28. Capitini CM, Herby S, Milliron M, Anver MR, Mackall CL, Fry TJ. Bone marrow deficient in IFN\{gamma\} signaling selectively reverses GVHD-associated immunosuppression and enhances a tumorspecific GVT effect. Blood. 2009;113(20):5002-5009.

29. McGill J, Legge KL. Cutting edge: contribution of lung-resident $\mathrm{T}$ cell proliferation to the overall magnitude of the antigen-specific CD8 T cell response in the lungs following murine influenza virus infection. J Immunol. 2009;183(7):4177-4181.

30. Yang YG, Dey BR, Sergio JJ, Pearson DA, Sykes M. Donor-derived interferon gamma is required for inhibition of acute graft-versus-host disease by interleukin 12. J Clin Invest. 1998;102(12):2126-2135.

31. Refaeli Y, Van Parijs L, Alexander SI, Abbas AK. Interferon gamma is required for activation-induced death of T lymphocytes. J Exp Med. 2002;196(7):999-1005.

32. Edinger M, et al. CD4+CD25+ regulatory T cells preserve graft-versus-tumor activity while inhibiting graft-versus-host disease after bone marrow transplantation. Nat Med. 2003;9(9):1144-1150.

33. Fondi $\mathrm{C}$, et al. Increase in FOXP3+ regulatory $\mathrm{T}$ cells in GVHD skin biopsies is associated with lower disease severity and treatment response. Biol Blood Marrow Transplant. 2009;15(8):938-947.

34. Nishibori T, Tanabe Y, Su L, David M. Impaired development of CD4+ CD25+ regulatory $\mathrm{T}$ cells in the absence of STAT1: increased susceptibility to autoimmune disease. J Exp Med. 2004;199(1):25-34.

35. Zhou L, Chong MM, Littman DR. Plasticity of CD4+ T cell lineage differentiation. Immunity. 2009; 30(5):646-655.

36. Marie JC, Letterio JJ, Gavin M, Rudensky AY. TGFbeta1 maintains suppressor function and Foxp3 expression in CD4+CD25+ regulatory T cells. J Exp Med. 2005;201(7):1061-1067.

37. Tamura T, et al. An IRF-1-dependent pathway of DNA damage-induced apoptosis in mitogen-activated T lymphocytes. Nature. 1995;376(6541):596-599.

38. Stephanou A, Latchman DS. Opposing actions of STAT-1 and STAT-3. Growth Factors. 2005; 23(3):177-182.

39. Tanabe Y, Nishibori T, Su L, Arduini RM, Baker DP, David M. Cutting edge: role of STAT1, STAT3, and STAT5 in IFN-alpha beta responses in T lymphocytes. JImmunol. 2005;174(2):609-613.

40. Wong LH, et al. Isolation and characterization of a human STAT1 gene regulatory element. Inducibility by interferon (IFN) types I and II and role of IFN regulatory factor-1. J Biol Chem. 2002; 277(22):19408-19417.

41. Fragale A, et al. IFN regulatory factor- 1 negatively regulates $\mathrm{CD} 4+\mathrm{CD} 25+$ regulatory $\mathrm{T}$ cell differentiation by repressing Foxp 3 expression. J Immunol. 2008;181(3):1673-1682.
42. Mapara MY, et al. Expression of chemokines in GVHD target organs is influenced by conditioning and genetic factors and amplified by GVHR. Biol Blood Marrow Transplant. 2006;12(6):623-634.

43. Duffner U, et al. Role of CXCR3-induced donor Tcell migration in acute GVHD. Exp Hematol. 2003; 31(10):897-902.

44. Shlomchik WD, et al. Prevention of graft versus host disease by inactivation of host antigen-presenting cells. Science. 1999;285(5426):412-415.

45. Lee CK, Smith E, Gimeno R, Gertner R, Levy DE. STAT1 affects lymphocyte survival and proliferation partially independent of its role downstream of IFN-gamma. J Immunol. 2000;164(3):1286-1292.

46. Qing Y, Stark GR. Alternative activation of STAT1 and STAT3 in response to interferon-gamma.J Biol Chem. 2004;279(40):41679-41685.

47. Lehtonen A, Matikainen S, Julkunen I. Interferons up-regulate STAT1, STAT2, and IRF family transcription factor gene expression in human peripheral blood mononuclear cells and macrophages. J Immunol. 1997;159(2):794-803

48. Saha B, Jyothi Prasanna S, Chandrasekar B, Nandi D. Gene modulation and immunoregulatory roles of Interferongamma. Cytokine. 2010;50(1):1-14.

49. Choudhury GG. A linear signal transduction pathway involving phosphatidylinositol 3-kinase, protein kinase Cepsilon, and MAPK in mesangial cells regulates interferon-gamma-induced STAT1alpha transcriptional activation. J Biol Chem. 2004; 279(26):27399-27409.

50. Pallandre JR, et al. Role of STAT3 in CD4+CD25+ FOXP3+ regulatory lymphocyte generation: implications in graft-versus-host disease and antitumor immunity. J Immunol. 2007;179(11):7593-7604.

51. Bettelli E, Sullivan B, Szabo SJ, Sobel RA, Glimcher LH, Kuchroo VK. Loss of T-bet, but not STAT1, prevents the development of experimental autoimmune encephalomyelitis. J Exp Med. 2004;200(1):79-87.

52. Caretto D, Katzman SD, Villarino AV, Gallo E, Abbas AK. Cutting edge: the Th1 response inhibits the generation of peripheral regulatory $\mathrm{T}$ cells. J Immunol. 2010;184(1):30-34.

53. Chang JH, Kim YJ, Han SH, Kang CY. IFN-gammaSTAT1 signal regulates the differentiation of inducible Treg: potential role for ROS-mediated apoptosis. Eur Immunol. 2009;39(5):1241-1251.

54. Thompson JS, Chu Y, Glass JF, Brown SA. Absence of IL-23p19 in donor allogeneic cells reduces mortality from acute GVHD. Bone Marrow Transplant. 2010;45(4):712-722.

55. Kappel LW, et al. IL-17 contributes to CD4mediated graft-versus-host disease. Blood. 2009; 113(4):945-952.

56. Yi T, et al. Absence of donor Th17 leads to augmented Th1 differentiation and exacerbated acute graftversus-host disease. Blood. 2008;112(5):2101-2110.

57. Bai Y, et al. Interferon-gamma induces X-linked inhibitor of apoptosis-associated factor- 1 and Noxa expression and potentiates human vascular smooth muscle cell apoptosis by STAT3 activation. J Biol Chem. 2008;283(11):6832-6842.

58. Meraz MA, et al. Targeted disruption of the Stat1 gene in mice reveals unexpected physiologic specificity in the JAK-STAT signaling pathway. Cell. 1996; 84(3):431-442.

59. Durbin JE, Hackenmiller R, Simon MC, Levy DE. Targeted disruption of the mouse Stat 1 gene results in compromised innate immunity to viral disease. Cell. 1996;84(3):443-450.

60. Shresta S, Sharar KL, Prigozhin DM, Snider HM, Beatty PR, Harris E. Critical roles for both STAT1dependent and STAT1-independent pathways in the control of primary dengue virus infection in mice. J Immunol. 2005;175(6):3946-3954.

61. Johnson LM, Scott P. STAT1 expression in dendritic cells, but not $\mathrm{T}$ cells, is required for immunity to Leishmania major. JImmunol. 2007;178(11):7259-7266. 
62. Mott KR, Underhill D, Wechsler SL, Town T, Ghiasi $\mathrm{H}$. A role for the JAK-STAT1 pathway in blocking replication of HSV-1 in dendritic cells and macrophages. Virol J. 2009;6:56.

63. Quigley M, Huang X, Yang Y. STAT1 signaling in CD8 T cells is required for their clonal expansion and memory formation following viral infection in vivo. J Immunol. 2008;180(4):2158-2164.

64. Fallarino F, Gajewski TF. Cutting edge: differentiation of antitumor CTL in vivo requires host expression of Stat1. J Immunol. 1999;163(8):4109-4113.
65. Pilz A, et al. Dendritic cells require STAT-1 phosphorylated at its transactivating domain for the induction of peptide-specific CTL. J Immunol. 2009; 183(4):2286-2293.

66. Fujita M, et al. Role of type 1 IFNs in antiglioma immunosurveillance--using mouse studies to guide examination of novel prognostic markers in humans. Clin Cancer Res. 2010;16(13):3409-3419.

67 . Hill GR, et al. Interleukin-11 promotes $\mathrm{T}$ cell polarization and prevents acute graft-versus-host disease after allogeneic bone marrow transplantation.
J Clin Invest. 1998;102(1):115-123.

68. Legge KL, Braciale TJ. Accelerated migration of respiratory dendritic cells to the regional lymph nodes is limited to the early phase of pulmonary infection. Immunity. 2003;18(2):265-277.

69. Lentzsch S, Gries M, Janz M, Bargou R, Dorken B, Mapara MY. Macrophage inflammatory protein 1-alpha (MIP-1 alpha ) triggers migration and signaling cascades mediating survival and proliferation in multiple myeloma (MM) cells. Blood. 2003; 101(9):3568-3573. 